\title{
On extinction of infectious diseases for multi-group SIRS models with saturated incidence rate
}

\author{
Qixing $\operatorname{Han}^{1}$ and Zhigang Wang ${ }^{2,3 *}$
}

\author{
"Correspondence: \\ wangzg2003205@163.com \\ 25chool of Mathematical Sciences, \\ Harbin Normal University, Harbin, \\ 150500, P.R. China \\ ${ }^{3}$ College of Mathematics, Jilin \\ University, Changchun, 130012 \\ P.R. China \\ Full list of author information is \\ available at the end of the article
}

\begin{abstract}
We generalize the deterministic and the stochastic single-group SIRS epidemic models with saturated incidence rate introduced by Lahrouz, Omari, and Kiouach to the multi-group versions. In the deterministic multi-group model, the fact is highlighted that if the threshold $\mathscr{R}_{0} \leq 1$, then the infective condition disappears and it means the extinction of the disease. If $\mathscr{R}_{0}>1$, then there exists an endemic equilibrium in a feasible region. Allowing the noise perturbation, for the stochastic version, we utilize stochastic Lyapunov functions to show the stability of the disease-free equilibrium of system. A detailed analysis is performed on almost surely exponential stability and $p$ th moment exponential stability of the disease-free equilibrium. We also go into several numerical simulations to illustrate how exactly the theoretical results are verified. Good agreement was observed between our theoretical results and numerical simulations. A comprehensive conclusion is provided.
\end{abstract}

Keywords: multi-group SIRS model; stochastic perturbation; saturated incidence rate; Brownian motion

\section{Introduction}

Epidemiology models have been widely studied by many mathematicians and biologists [1-7]. Most of the single-group models were considered in previous studies. Considering a heterogeneous population whose individuals are distinguishable by age, geography, and/or stage of disease and so on, it will be more realistic and reasonable to divide the individual hosts into groups. Therefore, many mathematicians devote themselves to realistic multi-group models for the spreading dynamics of infections. For example, Lajmanovich and Yorke in [4] have established a multi-group model for gonorrhea in a heterogeneous population where recovery is not immunized. Subsequently, this lead to increased investigations of the multi-group model; see [3, 5-8]. Considering the effect of nonlinear incidence rates for some disease transmissions, Capasso and Serio in [9] have put forward the saturated incidence rate taking the form $\frac{\beta S I}{1+a I}$ when they studied the cholera epidemic spread in Bari in 1973. The crowding effect of the infective individuals can be well reflected by $\frac{1}{1+a I}$ and the infection force of the disease can be measured by $\beta I$. Concerning this factor, Lahrouz et al. also incorporated the saturated incidence rate into deterministic and stochastic SIRS epidemic models [10]. The deterministic system formulated by them is as

(c) 2015 Han and Wang. This article is distributed under the terms of the Creative Commons Attribution 4.0 International License (http://creativecommons.org/licenses/by/4.0/), which permits unrestricted use, distribution, and reproduction in any medium, provided you give appropriate credit to the original author(s) and the source, provide a link to the Creative Commons license, and indicate if changes were made. 
Table 1 Summary of notation

\begin{tabular}{ll}
\hline Notation & Explanation \\
\hline$\beta_{k j}$ & Rate of disease transmission between compartments $S_{k}$ and $l_{j}$ \\
$d_{k^{\prime}}^{S}, d_{k^{\prime}}^{\prime} d_{k}^{R}$ & Nature mortality of $S, I, R$ compartments in the $k$ th group, respectively \\
$\Lambda_{k}$ & Recruitment rate of the population into the $k$ th group \\
$\varepsilon_{k}$ & Fatality rate in the $k$ th group \\
$\gamma_{k}$ & Recovery rate of infected individuals in the $k$ th group \\
$\delta_{k}$ & Removed rate of recovered individuals in the $k$ th group \\
$\alpha_{k}$ & Positive parameter related to $k$ th group \\
\hline
\end{tabular}

follows:

$$
\left\{\begin{array}{l}
\frac{\mathrm{d} S}{\mathrm{~d} t}=b-\mu S-\frac{\beta S I}{1+a I}+\gamma R \\
\frac{\mathrm{d} I}{\mathrm{~d} t}=-(\mu+c+\alpha) I+\frac{\beta S I}{1+a I} \\
\frac{\mathrm{d} R}{\mathrm{~d} t}=-(\mu+\gamma) R+\alpha I
\end{array}\right.
$$

Correspondingly, the stochastic model is as follows [10]:

$$
\left\{\begin{array}{l}
\mathrm{d} S=\left[b-\mu S-\frac{\beta S I}{1+a I}+\gamma R\right] \mathrm{d} t-\sigma \frac{S I}{1+a I} \mathrm{~d} B \\
\mathrm{~d} I=\left[-(\mu+c+\alpha) I+\frac{\beta S I}{1+a I}\right] \mathrm{d} t+\sigma \frac{S I}{1+a I} \mathrm{~d} B \\
\mathrm{~d} R=[-(\mu+\gamma) R+\alpha I] \mathrm{d} t .
\end{array}\right.
$$

We put forward the multi-group version of the deterministic equation (1.1).

$$
\left\{\begin{array}{l}
\mathrm{d} S_{k}(t)=\left[\Lambda_{k}-d_{k}^{S} S_{k}(t)-\sum_{j=1}^{n} \frac{\beta_{k j} S_{k}(t) I_{j}(t)}{1+\alpha_{k} I_{j}(t)}+\delta_{k} R_{k}(t)\right] \mathrm{d} t, \\
\mathrm{~d} I_{k}(t)=\left[\sum_{j=1}^{n} \frac{\beta_{k} S_{k}(t) I_{j}(t)}{1+\alpha_{k} I_{j}(t)}-\left(d_{k}^{I}+\gamma_{k}+\epsilon_{k}\right) I_{k}(t)\right] \mathrm{d} t, \\
\mathrm{~d} R_{k}(t)=\left[\gamma_{k} I_{k}(t)-\left(d_{k}^{R}+\delta_{k}\right) R_{k}(t)\right] \mathrm{d} t, \quad k=1,2, \ldots, n,
\end{array}\right.
$$

here $S_{k}(t), I_{k}(t)$, and $R_{k}(t)$ represent the number of susceptible, infective, and recovered individuals of the $k$ th group at time $t$, respectively. We summarize the parameters of system (1.3) in Table 1.

It is assumed that all parameters are positive throughout this paper. Ideally, the parameters involved in ecological models are constant. With this understanding, one only needs to consider the deterministic models. However, in reality, some parameters will be subject to the influence of fluctuating environment inevitably. In other words, some data derived from the environment are not constant but fluctuate around some average value due to continuous fluctuations in the environment. With this in mind, some researchers try to introduce perturbations into epidemic models. There exist two kinds of main perturbation approaches in terms of containing the random effects in models. One is to perturb the positive endemic equilibria in order for making robust the equilibria of deterministic models. In this situation, the essence of the investigation using the approach is to check if the asymptotic stability of the positive equilibria of deterministic models can be preserved. For example, Shaikhet in [2] proved the stability of the positive equilibrium for a predator-prey model with delays and stochastic perturbations. We in [11] discussed the global stability of the multi-group SEIQR model with random perturbation around the positive equilibrium in computer network. In addition, we also applied this approach to other epidemic models $[12,13]$. The other important approach is with parameters perturbation. Much literature on this approach can be found, such as $[7,14-16]$. In this paper, we 
adopt the second approach to endow the stochastic form of the deterministic model (1.3) and introduce randomness by replacing the parameters $\beta_{k k}$ by $\beta_{k k}+\sigma_{k} \frac{\mathrm{d} B_{k}}{\mathrm{~d} t}$, where $B_{k}(t)$, $k=1,2, \ldots, n$, are mutual independent standard Brownian motions with $B_{k}(0)=0$, and $\sigma_{k}$, $k=1,2, \ldots, n$ are the intensities of the white noises. We consider the stochastic version of (1.3) formulated by the equations:

$$
\left\{\begin{array}{l}
\mathrm{d} S_{k}(t)=\left[\Lambda_{k}-d_{k}^{S} S_{k}(t)-\sum_{j=1}^{n} \frac{\beta_{k} S_{k}(t) I_{j}(t)}{1+\alpha_{k} I_{j}(t)}+\delta_{k} R_{k}(t)\right] \mathrm{d} t-\sigma_{k} \frac{S_{k}(t) I_{k}(t)}{1+\alpha_{k} I_{k}(t)} \mathrm{d} B_{k}(t), \\
\mathrm{d} I_{k}(t)=\left[\sum_{j=1}^{n} \frac{\beta_{k j} S_{k}(t) I_{j}(t)}{1+\alpha_{k} I_{j}(t)}-\left(d_{k}^{I}+\gamma_{k}+\epsilon_{k}\right) I_{k}(t)\right] \mathrm{d} t+\sigma_{k} \frac{S_{k}(t) I_{k}(t)}{1+\alpha_{k} I_{k}(t)} \mathrm{d} B_{k}(t), \\
\mathrm{d} R_{k}(t)=\left[\gamma_{k} I_{k}(t)-\left(d_{k}^{R}+\delta_{k}\right) R_{k}(t)\right] \mathrm{d} t, \quad k=1,2, \ldots, n .
\end{array}\right.
$$

Our overall aim here is to discuss the extinction not only for a deterministic system but also for a stochastic system. In the course of using the Lyapunov analysis methods, the main difficulty for a multi-dimensional stochastic system is how we can effectively tackle drift and diffusion terms. The remaining sections are arranged as follows. Section 2 has the important result of Theorem 2.3 for deterministic model (1.3). Globally asymptotic stability of the disease-free equilibrium for deterministic (1.3) is demonstrated when $\mathscr{R}_{0} \leq 1$, which implies the extinction of the disease. The most significant results for the current study are proposed in Section 3. After choosing a key stochastic Lyapunov function, we verify the disease-free equilibrium $P_{0}$ of (1.4) is $p$ th moment exponentially stable at large and the disease-free equilibrium $P_{0}$ is almost surely exponentially stable under a suitable condition. By means of numerical methods, we give a dynamical analysis of (1.4) in Section 4. In the following section, a comprehensive conclusion is provided. Taking into account the biological meaning, in this paper, we always assume that

$$
d_{k}^{S} \leq \min \left\{d_{k}^{I}, d_{k}^{R}\right\}
$$

\section{Deterministic multi-group SIRS model with saturated incidence rate}

Extinction is one of the most important concerns in the course of studying infectiousdisease models. The substantive and core work is refined to one involving the asymptotical stability at large of the disease-free equilibrium. In this section, we devote ourselves to the deterministic equation (1.3). The objective is to find suitable conditions determining the extinction of disease for (1.3). In addition, a sufficient criterion is established for the asymptotical stability at large of the disease-free equilibrium of (1.3). We briefly mention an existence result for the endemic equilibrium of (1.3).

There always exists the unique disease-free equilibrium $P_{0}$ for (1.3),

$$
P_{0}=\left(S_{1}^{0}, 0,0, S_{2}^{0}, 0,0, \ldots, S_{n}^{0}, 0,0\right)
$$

where

$$
S_{k}^{0}=\frac{\Lambda_{k}}{d_{k}^{S}}, \quad k=1,2, \ldots, n .
$$

We can check the solution of (1.3) with initial condition $\left(S_{1}(0), I_{1}(0), R_{1}(0), \ldots, S_{n}(0), I_{n}(0)\right.$, $\left.R_{n}(0)\right) \in R_{+}^{3 n}$ remains nonnegative. Thus we only consider system (1.3) in $R_{+}^{3 n}$. Adding the 
several equations of system (1.3) together for every $k$ and in combination with the inequality above, we have

$$
\mathrm{d}\left(S_{k}+I_{k}+R_{k}\right) \leq\left(\Lambda_{k}-d_{k}^{S}\left(S_{k}+I_{k}+R_{k}\right)\right) \mathrm{d} t
$$

Hence, sup $\lim _{t \rightarrow \infty}\left(S_{k}+I_{k}+R_{k}\right) \leq \frac{\Lambda_{k}}{d_{k}^{s}}$. Given the bounded region of $R^{3 n}$ by

$$
\begin{aligned}
\Gamma= & \left\{\left(S_{1}, I_{1}, R_{1}, \ldots, S_{n}, I_{n}, R_{n}\right) \in R^{3 n} \mid 0 \leq S_{k} \leq \frac{\Lambda_{k}}{d_{k}^{S}}, 0 \leq I_{k} \leq \frac{\Lambda_{k}}{d_{k}^{S}}, 0 \leq R_{k} \leq \frac{\Lambda_{k}}{d_{k}^{S}},\right. \\
& \left.S_{k}+I_{k}+R_{k} \leq \frac{\Lambda_{k}}{d_{k}^{S}}, 1 \leq k \leq n\right\} .
\end{aligned}
$$

Omega limit sets of (1.3) are contained in $\Gamma$. $\Gamma$ is a positive invariant of (1.3).

Let the interior of $\Gamma$ be

$$
\stackrel{\circ}{\Gamma}=\left\{\left(S_{1}, I_{1}, R_{1}, \ldots, S_{n}, I_{n}, R_{n}\right) \in R^{3 n} \mid 0<S_{k}, I_{k}, R_{k}, S_{k}+I_{k}+R_{k}<\frac{\Lambda_{k}}{d_{k}^{S}}, 1 \leq k \leq n\right\} .
$$

Set

$$
\mathscr{F}=\left(\begin{array}{ccc}
\beta_{11} S_{1}^{0} & \cdots & \beta_{1 n} S_{1}^{0} \\
\vdots & \ddots & \vdots \\
\beta_{n 1} S_{n}^{0} & \cdots & \beta_{n n} S_{n}^{0}
\end{array}\right)
$$

and

$$
\mathscr{V}=\operatorname{diag}\left(d_{k}+\gamma_{k}+\epsilon_{k}\right)=\left(\begin{array}{cccc}
d_{1}+\gamma_{1}+\epsilon_{1} & 0 & \cdots & 0 \\
0 & d_{2}+\gamma_{2}+\epsilon_{2} & \cdots & 0 \\
\vdots & \vdots & \ddots & \vdots \\
0 & 0 & \cdots & d_{n}+\gamma_{n}+\epsilon_{n}
\end{array}\right)
$$

Then the next generation matrix is

$$
\mathscr{F} \mathscr{V}^{-1}=\left(\begin{array}{ccc}
\frac{\beta_{11} S_{1}^{0}}{d_{1}+\gamma_{1}+\epsilon_{1}} & \cdots & \frac{\beta_{1 n} S_{1}^{0}}{d_{n}+\gamma_{n}+\epsilon_{n}} \\
\vdots & \ddots & \vdots \\
\frac{\beta_{n 1} S_{n}^{0}}{d_{1}+\gamma_{1}+\epsilon_{1}} & \cdots & \frac{\beta_{n n} S_{n}^{0}}{d_{n}+\gamma_{n}+\epsilon_{n}}
\end{array}\right)
$$

The reproduction number of $(1.3)$ is

$$
\mathscr{R}_{0}=\rho\left(\mathscr{F} \mathscr{V}^{-1}\right)=\max \left\{|\lambda| ; \lambda \in \sigma\left(\mathscr{F} \mathscr{V}^{-1}\right)\right\}
$$

where $\sigma$ and $\rho$ denote the set of eigenvalues of a matrix and the spectral radius, respectively. Furthermore, the following proposition will be stated [8, 17].

Proposition 2.1 ([8,17]) For system (1.3), the disease-free equilibrium $P_{0}$ is locally asymptotically stable if $\mathscr{R}_{0}<1$ while it is unstable if $\mathscr{R}_{0}>1$. 
Let

$$
\mathscr{V}^{-1} \mathscr{F}=\left(\begin{array}{ccc}
\frac{\beta_{11} S_{1}^{0}}{d_{1}+\gamma_{1}+\epsilon_{1}} & \cdots & \frac{\beta_{1 n} S_{1}^{0}}{d_{1}+\gamma_{1}+\epsilon_{1}} \\
\vdots & \ddots & \vdots \\
\frac{\beta_{n 1} S_{n}^{0}}{d_{n}+\gamma_{n}+\epsilon_{n}} & \cdots & \frac{\beta_{n n} S_{n}^{0}}{d_{n}+\gamma_{n}+\epsilon_{n}}
\end{array}\right) .
$$

Following $[3,5,8]$, the threshold property of spectral radius of $\mathscr{V}^{-1} \mathscr{F}$ is similar to that of $\mathscr{F} \mathscr{V}^{-1}$. The following lemma can be stated immediately.

Lemma $2.2([3,5,8]) \rho\left(\mathscr{V}^{-1} \mathscr{F}\right) \leq 1$ is equivalent to $\mathscr{R}_{0} \leq 1$.

Furthermore, we state that the following.

Theorem 2.3 Assume $B=\left(\beta_{k j}\right)$ is irreducible. If $\mathscr{R}_{0} \leq 1$, then the disease-free equilibrium $P_{0}$ of (1.3) is globally asymptotically stable in $\Gamma$ and there is no endemic equilibrium $P^{*}$ at all in $\Gamma$.

Proof Note that $\rho\left(\mathscr{V}^{-1} \mathscr{F}\right) \leq 1$ from Lemma 2.2. Following [3, 5], define a matrix-valued function

$$
\mathcal{M}(\mathbf{S}, \mathbf{I})=\left(\begin{array}{ccc}
\frac{\beta_{11} S_{1}}{\left(d_{1}+\gamma_{1}+\epsilon_{1}\right)\left(1+\alpha_{1} I_{1}\right)} & \cdots & \frac{\beta_{1 n} S_{1}}{\left(d_{1}+\gamma_{1}+\epsilon_{1}\right)\left(1+\alpha_{1} I_{n}\right)} \\
\vdots & \ddots & \vdots \\
\frac{\beta_{n !} S_{n}}{\left(d_{n}+\gamma_{n}+\epsilon_{n}\right)\left(1+\alpha_{n} I_{1}\right)} & \cdots & \frac{\beta_{n 1} S_{n}}{\left(d_{n}+\gamma_{n}+\epsilon_{n}\right)\left(1+\alpha_{n} I_{n}\right)}
\end{array}\right)
$$

on $R_{+}^{3 n}$, where $\mathbf{S}=\left(S_{1}, \ldots, S_{n}\right)^{T}, \mathbf{I}=\left(I_{1}, \ldots, I_{n}\right)^{T}$. First, it can be claimed that there is no any endemic equilibrium $P^{*}$ in $\Gamma$. Because $\alpha_{j}>0$, we have $0<\mathcal{M}(\mathbf{S}, \mathbf{I})<\mathscr{V}^{-1} \mathscr{F}$. Since the nonnegative matrix $\mathcal{M}(\mathbf{S}, \mathbf{I})+\mathscr{V}^{-1} \mathscr{F}$ is irreducible, it follows from the Perron-Frobenius theorem (see [18]) that $\rho(\mathcal{M}(\mathbf{S}, \mathbf{I}))<\rho\left(\mathscr{V}^{-1} \mathscr{F}\right) \leq 1$. This implies that the equation $\mathcal{M}(\mathbf{S}, \mathbf{I}) \mathbf{I}=\mathbf{I}$ has only the trivial solution $\mathbf{I}=0$, where $\mathbf{I}=\left(I_{1}, \ldots, I_{n}\right)^{T}$. Hence the claim is true.

Second, one can claim that $P_{0}$ is globally asymptotically stable in $\Gamma$. Note that the nonnegative irreducible matrix $\mathscr{V}^{-1} \mathscr{F}$ has a strictly positive left eigenvector $\ell:=\left(\ell_{1}, \ldots, \ell_{n}\right) \geq$ 0 belonging to the eigenvalue $\rho\left(\mathscr{V}^{-1} \mathscr{F}\right)[18]$. Define the operator

$$
L(\mathbf{I})=\sum_{i=1}^{n} \ell_{i} \frac{I_{i}}{d_{i}+\gamma_{i}+\epsilon_{i}}
$$

on $R_{+}^{n}$. Furthermore, we have the differential operator

$$
\begin{aligned}
L^{\prime}(\mathbf{I}) & =\sum_{i=1}^{n} \ell_{i}\left(\frac{S_{i} \sum_{j=1}^{n} \frac{\beta_{i j} I_{j}}{\left(1+\alpha_{i} I_{j}\right)}}{d_{i}+\gamma_{i}+\epsilon_{i}}-I_{i}\right) \\
& =\ell \cdot\left(\mathcal{M}(\mathbf{S}, \mathbf{I})-\mathbf{E}_{n}\right) \mathbf{I} \\
& \leq \ell \cdot\left(\mathscr{V}^{-1} \mathscr{F}-\mathbf{E}_{n}\right) \mathbf{I} \\
& =\ell \cdot\left(\rho\left(\mathscr{V}^{-1} \mathscr{F}\right)-1\right) \mathbf{I},
\end{aligned}
$$


where $\mathbf{E}_{n}$ and denote the $n \times n$ identity matrix and the inner product of vectors, respectively. If $\rho\left(\mathscr{V}^{-1} \mathscr{F}\right) \leq 1$, then $\ell \cdot\left(\rho\left(\mathscr{V}^{-1} \mathscr{F}\right)-1\right) \mathbf{I} \leq 0$. If $\rho\left(\mathscr{V}^{-1} \mathscr{F}\right)<1$. Then $L^{\prime}(\mathbf{I})=0$ is equivalent to $\mathbf{I}=0$. If $\rho\left(\mathscr{V}^{-1} \mathscr{F}\right)=1$, then it follows from (2.3) that $L^{\prime}(\mathbf{I})=0$ implies

$$
\ell \cdot \mathcal{M}(\mathbf{S}, \mathbf{I})=\ell \cdot \mathbf{I} \text {. }
$$

Because $0<\mathcal{M}(\mathbf{S}, \mathbf{I})<\mathscr{V}^{-1} \mathscr{F}$, therefore $\ell \cdot \mathcal{M}(\mathbf{S}, \mathbf{I})<\ell \cdot\left(\mathscr{V}^{-1} \mathscr{F}\right)=\rho\left(\mathscr{V}^{-1} \mathscr{F}\right) \ell=\ell$. Thus $\mathbf{I}=0$ is the unique solution of (2.4). In summary, whether $\rho\left(\mathscr{V}^{-1} \mathscr{F}\right)<1$ or $\rho\left(\mathscr{V}^{-1} \mathscr{F}\right)=1$, $L^{\prime}(\mathbf{I})=0$ is equivalent to $\mathbf{I}=0, \mathbf{I}=0$ is the unique fixed point of the differential operator $L^{\prime}$. It's also worth noting that if $\mathbf{I}=0$, then $(\mathbf{S}, \mathbf{R})=\left(\mathbf{S}_{0}, \mathbf{0}\right)$, where $\mathbf{S}_{0}=\left(\frac{\Lambda_{1}}{d_{1}^{S}}, \ldots, \frac{\Lambda_{n}}{d_{n}^{S}}\right)$. Hence the compact invariant subset of the set where $L^{\prime}(\mathbf{I})=0$ is only the singleton $\left\{P_{0}\right\} \subset \Gamma$. It readily follows from the La Salle invariance principle [19] that the second claim holds.

The proof of the following proposition and corollary for (1.3) is standard [17, 20, 21].

Proposition 2.4 Assume $B=\left(\beta_{k j}\right)$ is irreducible. If $\mathscr{R}_{0}>1$, then $P_{0}$ is unstable and (1.3) is uniformly persistent in $\stackrel{\circ}{\Gamma}$.

Corollary 2.5 Assume $B=\left(\beta_{k j}\right)$ is irreducible. If $\mathscr{R}_{0}>1$, then (1.3) has at least one endemic equilibrium.

\section{Perturbed multi-group SIRS model with saturated incidence rate}

\subsection{Existence and uniqueness of nonnegative solutions for stochastic model}

Theorem 3.1 There exists a unique solution $\left(S_{1}(t), I_{1}(t), R_{1}(t), \ldots, S_{n}(t), I_{n}(t), R_{n}(t)\right)$ of $(1.4)$ on $t \geq 0$ for any initial value $\left(S_{1}(0), I_{1}(0), R_{1}(0), \ldots, S_{n}(0), I_{n}(0), R_{n}(0)\right) \in \mathbb{R}_{+}^{3 n}$, and the solution $\left(S_{1}(t), I_{1}(t), R_{1}(t), \ldots, S_{n}(t), I_{n}(t), R_{n}(t)\right) \in \mathbb{R}_{+}^{3 n}$ for $t \in[0, \infty)$ almost surely.

Proof Given arbitrarily initial value $\left(S_{1}(0), I_{1}(0), R_{1}(0), \ldots, S_{n}(0), I_{n}(0), R_{n}(0)\right) \in \mathbb{R}_{+}^{3 n}$, because of local Lipschitz continuity of the coefficients of (1.4), there must be a unique local solution $\left(S_{1}(t), I_{1}(t), R_{1}(t), \ldots, S_{n}(t), I_{n}(t), R_{n}(t)\right)$ on $t \in\left[0, \tau_{e}\right)$, where $\tau_{e}$ is the explosion time (see $[10,22])$. To show the global existence of this solution, it must be claimed that $\tau_{e}=\infty$ a.s. Let $m_{0} \geq 0$ be sufficiently large so that $S_{k}(0), I_{k}(0), R_{k}(0)(k=1,2, \ldots, n)$ all lie within the interval $\left[\frac{1}{m_{0}}, m_{0}\right]$. For each integer $m \geq m_{0}$, define the stopping time

$$
\begin{aligned}
\tau_{m}= & \inf \left\{t \in\left[0, \tau_{e}\right): \min \left\{S_{k}(t), I_{k}(t), R_{k}(t), k=1, \ldots, n\right\} \leq \frac{1}{m}\right. \text { or } \\
& \left.\max \left\{S_{k}(t), I_{k}(t), R_{k}(t), k=1, \ldots, n\right\} \geq m\right\} .
\end{aligned}
$$

We set $\inf \emptyset=\infty$ (as usual $\emptyset$ denotes the empty set). It is obvious $\tau_{m}$ is increasing as $m \rightarrow \infty$. Set $\tau_{\infty}=\lim _{m \rightarrow \infty} \tau_{m}$, whence $\tau_{\infty} \leq \tau_{e}$ a.s. If we can show that $\tau_{\infty}=\infty$ a.s., then $\tau_{e}=\infty$ and $\left(S_{1}(t), I_{1}(t), R_{1}(t), \ldots, S_{n}(t), I_{n}(t), R_{n}(t)\right) \in \mathbb{R}_{+}^{3 n}$ a.s. for all $t \geq 0$. In other words, to complete the proof all we need to show is that $\tau_{\infty}=\infty$ a.s.

For $t \leq \tau_{m}$, we can see, for each $k$,

$$
\begin{aligned}
\mathrm{d}\left(S_{k}+I_{k}+R_{k}\right) & =\left(\Lambda_{k}-d_{k}^{S} S_{k}-d_{k}^{I} I_{k}-\epsilon_{k} I_{k}-d_{k}^{R} R_{k}\right) \mathrm{d} t \\
& \leq\left(\Lambda_{k}-d_{k}^{S}\left(S_{k}+I_{k}+R_{k}\right)\right) \mathrm{d} t
\end{aligned}
$$




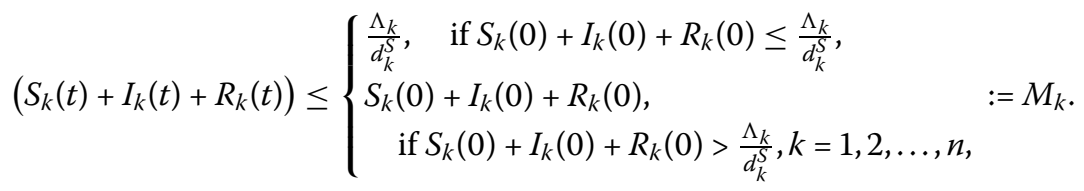

Define a $C^{2}$-function $V: R_{+}^{3 n} \rightarrow R_{+}$by

$$
V\left(S_{1}, I_{1}, R_{1}, \ldots, S_{n}, I_{n}, R_{n}\right)=\sum_{k=1}^{n}\left(\left(S_{k}-1-\ln S_{k}\right)+\left(I_{k}-1-\ln I_{k}\right)+\left(R_{k}-1-\ln R_{k}\right)\right) .
$$

Reviewing Ito's formula, for $s \in[0, \infty), t \in\left[0, s \wedge \tau_{m}\right]$, it can be derived that

$$
\begin{aligned}
\mathrm{d}(V(t))= & \sum_{k=1}^{n}\left[\left(1-\frac{1}{S_{k}}\right)\left(\Lambda_{k}-d_{k}^{S} S_{k}-\sum_{j=1}^{n} \frac{\beta_{k j} S_{k} I_{j}}{1+\alpha_{k} I_{j}}+\delta_{k} R_{k}\right)+\frac{\sigma_{k}^{2} I_{k}^{2}}{2\left(1+\alpha_{k} I_{k}\right)^{2}}\right] \mathrm{d} t \\
& +\sum_{k=1}^{n}\left[\left(1-\frac{1}{I_{k}}\right)\left(\sum_{j=1}^{n} \frac{\beta_{k j} S_{k} I_{j}}{1+\alpha_{k} I_{j}}-\left(d_{k}^{I}+\gamma_{k}+\epsilon_{k}\right) I_{k}\right)+\frac{\sigma_{k}^{2} S_{k}^{2}}{2\left(1+\alpha_{k} I_{k}\right)^{2}}\right] \mathrm{d} t \\
& +\sum_{k=1}^{n}\left(1-\frac{1}{R_{k}}\right)\left(\gamma_{k} I_{k}-\left(d_{k}^{R}+\delta_{k}\right) R_{k}\right) \mathrm{d} t+\sum_{k=1}^{n} \frac{\sigma_{k}\left(I_{k}-S_{k}\right)}{1+\alpha_{k} I_{k}} \mathrm{~d} B(t) \\
= & \sum_{k=1}^{n}\left(\Lambda_{k}+d_{k}^{S}+d_{k}^{I}+\gamma_{k}+\epsilon_{k}+d_{k}^{R}+\delta_{k}\right. \\
& +\sum_{j=1}^{n} \frac{\beta_{k j} I_{j}}{\left(1+\alpha_{k} I_{j}\right)}-\sum_{j=1}^{n} \frac{\beta_{k j} S_{k} I_{j}}{\left(1+\alpha_{k} I_{j}\right)}-\frac{d_{k}}{S_{k}}\left(\frac{\Lambda_{k}}{d_{k}^{S}}-S_{k}\right) \\
& -\frac{\delta_{k} R_{k}}{S_{k}}-\frac{\gamma_{k} I_{k}}{R_{k}}-\left(d_{k}^{S}+\varepsilon_{k}\right) S_{k}-\left(d_{k}^{I}+\gamma_{k}+\epsilon_{k}\right) I_{k} \\
& \left.-\left(d_{k}^{R}+\delta_{k}\right) R_{k}+\frac{\sigma_{k}^{2}\left(S_{k}^{2}+I_{k}^{2}\right)}{2\left(1+\alpha_{k} I_{k}\right)^{2}}\right) \mathrm{d} t+\sum_{k=1}^{n} \frac{\sigma_{k}\left(I_{k}-S_{k}\right)}{1+\alpha_{k} I_{k}} \mathrm{~d} B(t) \\
\leq & \sum_{k=1}^{n}\left(\Lambda_{k}+d_{k}^{S}+\gamma_{k}^{I}+\epsilon_{k}+d_{k}^{R}+\delta_{k}+\sum_{j=1}^{n} \beta_{k j} M_{j}+\sigma_{k}^{2} M_{k}^{2}\right) \mathrm{d} t \\
& +\sum_{k=1}^{n} \frac{\sigma_{k}\left(I_{k}-S_{k}\right)}{1+\alpha_{k} I_{k}} \mathrm{~d} B(t) .
\end{aligned}
$$

Therefore,

$$
\mathrm{d}(V(t)) \leq N t+\sum_{k=1}^{n} \int_{0}^{t} \frac{\sigma_{k}\left(I_{k}-S_{k}\right)}{1+\alpha_{k} I_{k}} \mathrm{~d} t \quad \text { a.s. }
$$

where

$$
N=\sum_{k=1}^{n}\left(\Lambda_{k}+d_{k}^{S}+d_{k}^{I}+\gamma_{k}+\epsilon_{k}+d_{k}^{R}+\delta_{k}+\sum_{j=1}^{n} \beta_{k j} M_{j}+\sigma_{k}^{2} M_{k}^{2}\right) .
$$

If we take the expectation of the inequality above, then we obtain, for $s \in[0, \infty]$,

$$
E\left[V\left(S_{1}\left(\tau_{m} \wedge s\right)\right) \leq N s \wedge \tau_{m} \leq N s \quad\right. \text { a.s. }
$$


Set $\Omega_{m}=\left\{\tau_{m} \leq s\right\}$ for $m \geq m_{0}$. Note that for every $\omega \in \Omega_{m}$, there is at least one of $S_{k}\left(\tau_{m}, \omega\right), I_{k}\left(\tau_{m}, \omega\right)$, and $R_{k}\left(\tau_{m}, \omega\right), k=1, \ldots, n$ that equals either $m$ or $\frac{1}{m}$, and hence $V\left(S_{1}\left(\tau_{m}\right), I_{1}\left(\tau_{m}\right), R_{1}\left(\tau_{m}\right), \ldots, S_{n}\left(\tau_{m}\right), I_{n}\left(\tau_{m}\right), R_{n}\left(\tau_{m}\right)\right)$ is no less than $m-1-\ln m$ or $\frac{1}{m}-1-$ $\ln \frac{1}{m}=\frac{1}{m}-1+\ln m$. Consequently,

$$
\begin{aligned}
V\left(S_{1}\left(\tau_{m}\right), I_{1}\left(\tau_{m}\right), R_{1}\left(\tau_{m}\right), \ldots, S_{n}\left(\tau_{m}\right), I_{n}\left(\tau_{m}\right), R_{n}\left(\tau_{m}\right)\right) \\
\geq(m-1-\ln m) \wedge\left(\frac{1}{m}-1+\ln m\right), \\
\left.\left.\left.E\left[V\left(\tau_{m} \wedge s\right)\right)\right] \geq E\left[V\left(\tau_{m} \wedge s\right)\right)_{\chi_{\left(\tau_{m} \leq s\right)}}\right]+E\left[V\left(\tau_{m} \wedge s\right)\right)_{\chi_{\left(\tau_{m}>s\right)}}\right] \\
\quad \geq E\left[V\left(\tau_{m} \wedge s\right)\right)_{\left.\chi_{\left(\tau_{m} \leq s\right)}\right] .}
\end{aligned}
$$

It follows that

$$
\left.E\left[V\left(\tau_{m} \wedge s\right)\right)\right] \geq\left[(m-1-\ln m) \wedge\left(\frac{1}{m}-1+\ln m\right)\right] P\left(\tau_{m} \leq s\right) .
$$

Combining (3.2) with (3.5) gives for all $s \geq 0$

$$
P\left(\tau_{m} \leq s\right) \leq \frac{N s}{(m-1-\ln m) \wedge\left(\frac{1}{m}-1+\ln m\right)},
$$

where $1_{\Omega_{m}(\omega)}$ is the indicator function of $\Omega_{m}$. Letting $m \rightarrow \infty$, we get for $s \in[0, \infty]$, $P\left(\tau_{\infty} \leq s\right)=0$. Thus $P\left(\tau_{\infty}=\infty\right)=1$. So we must therefore have $\tau_{\infty}=\infty$ a.s.

Let

$$
\begin{aligned}
\Gamma^{*}= & \left\{\left(S_{1}, I_{1}, R_{1}, \ldots, S_{n}, I_{n}, R_{n}\right): S_{k}>0, I_{k} \geq 0, R_{k} \geq 0, S_{k}+I_{k}+R_{k} \leq \frac{\Lambda_{k}}{d_{k}^{S}},\right. \\
& k=1,2, \ldots, n, \text { almost surely }\} .
\end{aligned}
$$

Theorem 3.2 $\Gamma^{*}$ is a positively invariant set of (1.4), that is, if for any initial value $\left(S_{1}(0), I_{1}(0), R_{1}(0), \ldots, S_{n}(0), I_{n}(0), R_{n}(0)\right) \in \Gamma^{*}$, the solution $\left(S_{1}(t), I_{1}(t), R_{1}(t), \ldots, S_{n}(t), I_{n}(t)\right.$, $\left.R_{n}(t)\right) \in \Gamma^{*}$ of (1.4) for all $t \geq 0$ almost surely.

Proof Adding the equations of system (1.4) together for every $k \in\{1,2, \ldots, n\}$, we have

$$
\mathrm{d}\left(S_{k}(t)+I_{k}(t)+R_{k}(t)\right)=\left(\Lambda_{k}-d_{k}^{S} S_{k}(t)-d_{k}^{I} I_{k}(t)-\epsilon_{k} I_{k}(t)-d_{k}^{R} R_{k}(t)\right) \mathrm{d} t .
$$

Then, if $\left(S_{1}(s), \ldots, S_{n}(s), I(s), \ldots, I_{n}(s), R_{1}(s), \ldots, R_{n}(s)\right) \in R_{+}^{3 n}$ for $s \in[0, t]$ almost surely, we obtain

$$
\mathrm{d}\left(S_{k}(t)+I_{k}(t)+R_{k}(t)\right) \leq\left(\Lambda_{k}-d_{k}^{S}\left(S_{k}(t)-I_{k}(t)-R_{k}(t)\right)\right) \mathrm{d} t .
$$

By integration we check

$$
S_{k}(s)+I_{k}(s)+R_{k}(s) \leq \frac{\Lambda_{k}}{d_{k}^{S}}+e^{-d_{k}^{S} s}\left(S_{k}(0)+I_{k}(0)+R_{k}(0)-\frac{\Lambda_{k}}{d_{k}^{S}}\right) .
$$




$$
\begin{aligned}
& \text { If } S_{k}(0)+I_{k}(0)+R_{k}(0) \leq \frac{\Lambda_{k}}{d_{k}^{S}} \text {, then } S_{k}(s)+I_{k}(s)+R_{k}(s) \leq \frac{\Lambda_{k}}{d_{k}^{S}} \text { almost surely, thus } \\
& \qquad S_{k}(s), I_{k}(s), R_{k}(s) \in \Gamma^{*} \text { for } s \in[0, t] \text {, a.s. }
\end{aligned}
$$

This completes the proof.

The assumption that $\left(S_{1}(0), I_{1}(0), R_{1}(0), \ldots, S_{n}(0), I_{n}(0), R_{n}(0)\right) \in \Gamma^{*}$ will be used for the remaining parts of this paper.

\section{2 pth moment exponential stability at large and almost surely exponential stability in $\Gamma^{*}$}

Clearly, the disease-free equilibrium of (1.4) is $P_{0}=\left(\frac{\Lambda_{1}}{d_{1}^{S}}, 0,0, \ldots, \frac{\Lambda_{n}}{d_{n}^{S}}, 0,0\right)$. We have mentioned in Section 2 that when $\mathscr{R}_{0} \leq 1, P_{0}$ of (1.3) is globally stable. It means the extinction of disease will be inevitable after a period of time. Therefore, controlling the infectious disease by investigating the disease-free equilibrium $P_{0}$ of (1.4) is important as well. In this subsection, it will be verified that $P_{0}$ is $p$ th moment exponentially stable at large. Moreover, by using appropriate stochastic Lyapunov functions, almost surely exponential stability of $P_{0}$ also will be stated in this subsection.

Check the $d$-dimensional stochastic equation [23]

$$
\mathrm{d} \boldsymbol{x}(t)=f(\boldsymbol{x}(t), t) \mathrm{d} t+g(\boldsymbol{x}(t), t) \mathrm{d} B(t), \quad t \geq t_{0} .
$$

Provided that (3.6) satisfies the existence-and-uniqueness theorem, then, given arbitrarily initial value $\boldsymbol{x}\left(t_{0}\right)=\boldsymbol{x}_{0} \in \mathbb{R}^{d}$, there exists a unique global solution $\boldsymbol{x}\left(t ; t_{0}, \boldsymbol{x}_{0}\right)$ for (3.6). To study the stability, it will be assumed in this subsection that $f(\mathbf{0}, t)=0$ and $g(\mathbf{0}, t)=0$ for all $t \geq t_{0}$. So (3.6) admits a solution $\boldsymbol{x}(t) \equiv \mathbf{0}$, which is called an equilibrium position.

Denote by $C^{2,1}\left(\mathbb{R}^{d} \times\left[t_{0}, \infty\right) ; \mathbb{R}_{+}\right)$the family of all nonnegative functions $V(\boldsymbol{x}, t)$ on $\mathbb{R}^{d} \times\left[t_{0}, \infty\right)$ which are continuously twice differentiable in $\boldsymbol{x}$ and once in $t$. Define the differential operator $L$ associated with (3.6) by

$$
L=\frac{\partial}{\partial t}+\sum_{i=1}^{d} f_{i}(\boldsymbol{x}, t) \frac{\partial}{\partial x_{i}}+\frac{1}{2} \sum_{i, j=1}^{d}\left[g^{T}(\boldsymbol{x}, t) g(\boldsymbol{x}, t)\right]_{i j} \frac{\partial^{2}}{\partial x_{i} x_{j}} .
$$

Let $L$ act on a function $V \in C^{2,1}\left(\mathbb{R}^{d} \times\left[t_{0}, \infty\right) ; \mathbb{R}_{+}\right)$, then

$$
L V(\boldsymbol{x}, t)=V_{t}(\boldsymbol{x}, t)+V_{t}(\boldsymbol{x}, t) f(\boldsymbol{x}, t)+\frac{1}{2} \operatorname{trace}\left[g^{T}(\mathbf{x}, t) V_{x x}(\boldsymbol{x}, t) g(\boldsymbol{x}, t)\right]
$$

Definition 3.3 ([10, 23]) The equilibrium $X=0$ of the system (3.6) is said to be:

(1) stable in probability if for all $\epsilon>0$,

$$
\lim _{X_{0} \rightarrow 0} P\left(\sup _{t \geq 0}\left|X\left(t, X_{0}\right)\right| \geq \epsilon\right)=0
$$

(2) asymptotically stable if it is stable in probability and, moreover,

$$
\lim _{X_{0} \rightarrow 0} P\left(\lim _{t \rightarrow \infty} X\left(t, X_{0}\right)=0\right)=1
$$


(3) globally asymptotically stable if it is stable in probability and, moreover, for all $X_{0} \in \mathbb{R}^{d}$,

$$
P\left(\lim _{t \rightarrow \infty} X\left(t, X_{0}\right)=0\right)=1
$$

(4) almost surely exponentially stable if, for all $X_{0} \in \mathbb{R}^{d}$,

$$
\limsup _{t \rightarrow \infty} \frac{1}{t} \ln \left|X\left(t, X_{0}\right)\right|<0 \quad \text { a.s.; }
$$

(5) $p$ th moment exponentially stable if there is a pair of positive constants $C_{1}$ and $C_{2}$ such that, for all $X_{0} \in \mathbb{R}^{d}$,

$$
E\left(\left|X\left(t, X_{0}\right)\right|^{p}\right) \leq C_{1}\left|X_{0}\right|^{p} e^{-C_{2} t} \quad \text { on } t \geq 0
$$

Next we review the following lemma in which the conditions for the moment exponential stability of the equilibrium of (3.6) will be presented (see [10, 23]).

Lemma 3.4 Assume that there exists a function $V \in C^{2,1}\left(\mathbb{R}^{3 n} \times\left[t_{0}, \infty\right) ; \mathbb{R}_{+}\right)$such that the inequalities

$$
\begin{aligned}
& K_{1}|x|^{p} \leq V(x, t) \leq K_{2}|x|^{p}, \\
& L V(x, t) \leq-K_{3}|x|^{p}, \quad K_{i}>0, p>0,
\end{aligned}
$$

hold. Then the equilibrium of the system (3.6) is the pth moment exponentially stable. Usually, if $p=2$, we call it exponentially stable in mean square and the equilibrium $X=0$ is globally asymptotically stable.

As a special case of Young's inequality, the following lemma can be stated.

Lemma 3.5 Let $\varepsilon, x, y>0, p \geq 2$, then

$$
\begin{aligned}
& x^{p-1} y \leq \frac{(p-1) \varepsilon}{p} x^{p}+\frac{1}{p \varepsilon^{p-1}} y^{p}, \\
& x^{p-2} y^{2} \leq \frac{p-2}{p} \varepsilon x^{p}+\frac{2}{p} \varepsilon^{\frac{2-p}{2}} y^{p} .
\end{aligned}
$$

Theorem 3.6 Assume $B=\left(\beta_{k j}\right)_{n \times n}$ is irreducible and $p \geq 2$. If $\mathscr{R}_{0}=\rho\left(M_{0}\right)<1$ and $\left(d_{k}^{I}+\right.$ $\left.\gamma_{k}+\epsilon_{k}\right)-\sum_{j=1}^{n} \frac{\beta_{k j} \Lambda_{k}}{d_{k}^{S}}>\frac{p \Lambda_{k}^{2} \sigma_{k}^{2}}{2\left(d_{k}^{S}\right)^{2}}$ hold, then the disease-free equilibrium $P_{0}$ of $(1.4)$ is pth moment exponentially stable globally.

Proof Let $p \geq 2$ and $\left(S_{0}, I_{0}, R_{0}\right) \in \Gamma^{*}$, in view of Theorem 3.2 the solution of the system (1.4) remains in $\Gamma^{*}$. We know from the previous assertion that $M_{0}=\mathscr{V}^{-1} \mathscr{F}$ is irreducible, it follows that the nonnegative irreducible matrix $M_{0}$ has a strictly positive left eigenvector $\omega:=\left(\omega_{1}, \omega_{2}, \ldots, \omega_{n}\right)>0$ associated with the eigenvalue $\rho\left(M_{0}\right)$, such that $\left(\omega_{1}, \omega_{2}, \ldots, \omega_{n}\right) \rho\left(M_{0}\right)=\left(\omega_{1}, \omega_{2}, \ldots, \omega_{n}\right) M_{0}$. 
Han and Wang Advances in Difference Equations (2015) 2015:333

Page 11 of 20

Define a $C^{2}$-function $V: R_{+}^{3 n} \rightarrow R_{+}$by

$$
V\left(S_{1}, I_{1}, R_{1}, \ldots, S_{n}, I_{n}, R_{n}\right)=\sum_{k=1}^{n} a_{k}\left(\frac{\Lambda_{k}}{d_{k}^{S}}-S_{k}\right)^{p}+\frac{1}{p} \sum_{k=1}^{n} \frac{\omega_{k}}{d_{k}^{I}+\gamma_{k}+\epsilon_{k}} I_{k}^{p}+\sum_{k=1}^{n} b_{k} R_{k}^{p}
$$

where $a_{k}, b_{k}, k=1,2, \ldots, n$ are positive constants which we can choose in the following.

Set $V(\boldsymbol{x})=V_{1}(\boldsymbol{x})+V_{2}(\boldsymbol{x})+V_{3}(\boldsymbol{x})$, where

$$
V_{1}(\boldsymbol{x})=\sum_{k=1}^{n} a_{k}\left(\frac{\Lambda_{k}}{d_{k}^{S}}-S_{k}\right)^{p}, \quad V_{2}(\boldsymbol{x})=\frac{1}{p} \sum_{k=1}^{n} \frac{\omega_{k}}{d_{k}^{I}+\gamma_{k}+\epsilon_{k}} I_{k}^{p}, \quad V_{3}(\boldsymbol{x})=\sum_{k=1}^{n} b_{k} R_{k}^{p} .
$$

Denote $L$ as the generating operator of system (1.4). We calculate

$$
\begin{aligned}
L V_{1}= & -\sum_{k=1}^{n} p d_{k}^{S} a_{k}\left(\frac{\Lambda_{k}}{d_{k}^{S}}-S_{k}\right)^{p}+\sum_{k=1}^{n} \sum_{j=1}^{n} p \beta_{k j} a_{k} \frac{S_{k} I_{j}}{1+\alpha_{k} I_{j}}\left(\frac{\Lambda_{k}}{d_{k}^{S}}-S_{k}\right)^{p-1} \\
& -\sum_{k=1}^{n} \sum_{j=1}^{n} p \delta_{k} a_{k} R_{k}\left(\frac{\Lambda_{k}}{d_{k}^{S}}-S_{k}\right)^{p-1} \\
& +\frac{1}{2} \sum_{k=1}^{n} p(p-1) \sigma_{k}^{2} a_{k} \frac{S_{k}^{2} I_{K}^{2}}{\left(1+\alpha_{k} I_{k}\right)^{2}}\left(\frac{\Lambda_{k}}{d_{k}^{S}}-S_{k}\right)^{p-2}
\end{aligned}
$$

and

$$
\begin{aligned}
L V_{2}= & \sum_{k=1}^{n} \frac{\omega_{k}}{d_{k}^{I}+\gamma_{k}+\epsilon_{k}}\left(\sum_{j=1}^{n} \frac{\beta_{k j} S_{k} I_{k}^{p-1} I_{j}}{1+\alpha_{k} I_{j}}-\left(d_{k}^{I}+\gamma_{k}+\epsilon_{k}\right) I_{k}^{p}\right) \\
& +\frac{1}{2} \sum_{k=1}^{n}(p-1) \sigma_{k}^{2} \frac{S_{k}^{2} I_{k}^{p}}{\left(1+\alpha_{k} I_{k}\right)^{2}}, \\
L V_{3}= & \sum_{k=1}^{n} p b_{k} R_{k}^{p-1}\left(\gamma_{k} I_{k}-\left(d_{k}^{R}+\delta_{k}\right) R_{k}\right)=\sum_{k=1}^{n} p b_{k}\left(\gamma_{k} I_{k} R_{k}^{p-1}-\left(d_{k}^{R}+\delta_{k}\right) R_{k}^{p}\right),
\end{aligned}
$$

respectively. Because $S_{k}, I_{k}, R_{k} \in\left(0, \frac{\Lambda_{k}}{d_{k}^{S}}\right)$,

$$
\begin{aligned}
L V= & L V_{1}+L V_{2}+L V_{3} \\
\leq & -\sum_{k=1}^{n} p d_{k}^{S} a_{k}\left(\frac{\Lambda_{k}}{d_{k}^{S}}-S_{k}\right)^{p}+\sum_{k=1}^{n} \sum_{j=1}^{n} p \beta_{k j} a_{k} \frac{\Lambda_{k} I_{j}}{d_{k}^{S}}\left(\frac{\Lambda_{k}}{d_{k}^{S}}-S_{k}\right)^{p-1} \\
& +\frac{1}{2} \sum_{k=1}^{n} p(p-1) \sigma_{k}^{2} a_{k} \frac{\Lambda_{k}^{2} I_{k}^{2}}{\left(d_{k}^{S}\right)^{2}}\left(\frac{\Lambda_{k}}{d_{k}^{S}}-S_{k}\right)^{p-2} \\
& +\sum_{k=1}^{n} \frac{\omega_{k}}{d_{k}^{I}+\gamma_{k}+\epsilon_{k}}\left[\left(\frac{\sum_{j=1}^{n} \beta_{k j} \Lambda_{k} I_{k}^{p-1} I_{j}}{d_{k}^{S}}-\left(d_{k}^{I}+\gamma_{k}+\epsilon_{k}\right) I_{k}^{p}\right)+\frac{1}{2}(p-1) \sigma_{k}^{2} \frac{\Lambda_{k}^{2} I_{k}^{p}}{\left(d_{k}^{S}\right)^{2}}\right] \\
& +\sum_{k=1}^{n} p b_{k}\left(\gamma_{k} I_{k} R_{k}^{p-1}-\left(d_{k}^{R}+\delta_{k}\right) R_{k}^{p}\right) .
\end{aligned}
$$


Applying Lemma 3.5

$$
\begin{aligned}
& I_{k}^{p-1} I_{j} \leq \frac{p-1}{p} I_{k}^{p}+\frac{1}{p} I_{j}^{p}, \\
& I_{k}^{2}\left(\frac{\Lambda_{k}}{d_{k}^{S}}-S_{k}\right)^{p-2} \leq \frac{p-2}{p} \varepsilon\left(\frac{\Lambda_{k}}{d_{k}^{S}}-S_{k}\right)^{p}+\frac{2}{p} \varepsilon^{\frac{2-p}{2}} I_{k}^{p}, \\
& I_{j}\left(\frac{\Lambda_{k}}{d_{k}^{S}}-S_{k}\right)^{p-1} \leq \frac{p-1}{p} \varepsilon\left(\frac{\Lambda_{k}}{d_{k}^{S}}-S_{k}\right)^{p}+\frac{1}{p} \varepsilon^{1-p} I_{j}^{p} \\
& \leq \frac{p-1}{p} \varepsilon\left(\frac{\Lambda_{k}}{d_{k}^{S}}-S_{k}\right)^{p}+\frac{\left(\Lambda_{j}\right)^{p}}{p\left(d_{j}^{S}\right)^{p}} \varepsilon^{1-p}, \\
& I_{k} R_{k}^{p-1} \leq \frac{p-1}{p} \varepsilon R_{k}^{p}+\frac{1}{p} \varepsilon^{1-p} I_{k}^{p}, \\
& L V \leq-\sum_{k=1}^{n} a_{k}\left\{p d_{k}^{S}-\left[(p-1) \frac{\sum_{j=1}^{n} \beta_{k j} \Lambda_{k}}{d_{k}^{S}}+\frac{(p-1)(p-2) \sigma_{k}^{2} \Lambda_{k}^{2}}{2\left(d_{k}^{S}\right)^{2}}\right] \varepsilon\right\}\left(\frac{\Lambda_{k}}{d_{k}^{S}}-S_{k}\right)^{p} \\
& -\sum_{k=1}^{n} \omega_{k}\left[\left(1-\frac{(p-1) \sigma_{k}^{2} \Lambda_{k}^{2}}{2\left(d_{k}^{S}\right)^{2}\left(d_{k}^{I}+\gamma_{k}+\epsilon_{k}\right)}-\frac{\sum_{j=1}^{n} \beta_{k j}(p-1) \Lambda_{k}}{p\left(d_{k}^{I}+\gamma_{k}+\epsilon_{k}\right) d_{k}^{S}}\right) I_{k}^{p}\right. \\
& \left.-\frac{\sum_{j=1}^{n} \beta_{k j} \Lambda_{k}}{p d_{k}^{S}\left(d_{k}^{I}+\gamma_{k}+\epsilon_{k}\right)} I_{j}^{p}\right] \\
& +\sum_{k=1}^{n}\left[a_{k} \frac{(p-1) \sigma_{k}^{2} \Lambda_{k}^{2}}{\left(d_{k}^{S}\right)^{2}} \varepsilon^{\frac{2-p}{2}}+b_{k} \gamma_{k} \varepsilon^{1-p}\right] I_{k}^{p}+\sum_{k=1}^{n} a_{k} \frac{\sum_{j=1}^{n} \beta_{k j} \Lambda_{k}\left(\Lambda_{j}\right)^{p}}{d_{k}^{S}\left(d_{j}^{S}\right)^{p}} \varepsilon^{1-p} \\
& -\sum_{k=1}^{n} b_{k}\left(p\left(d_{k}^{R}+\delta_{k}\right)-(p-1) \varepsilon \gamma_{k}\right) R_{k}^{p}
\end{aligned}
$$

Because $\left(d_{k}^{I}+\gamma_{k}+\epsilon_{k}\right)-\sum_{j=1}^{n} \frac{\beta_{k j} \Lambda_{k}}{d_{k}^{S}}>\frac{p \Lambda_{k}^{2} \sigma_{k}^{2}}{2\left(d_{k}^{S}\right)^{2}}$,

$$
\begin{aligned}
& -\sum_{k=1}^{n} \omega_{k}\left(1-\frac{(p-1) \sigma_{k}^{2} \Lambda_{k}^{2}}{2\left(d_{k}^{S}\right)^{2}\left(d_{k}^{I}+\gamma_{k}+\epsilon_{k}\right)}-\frac{\sum_{j=1}^{n} \beta_{k j}(p-1) \Lambda_{k}}{p\left(d_{k}^{I}+\gamma_{k}+\epsilon_{k}\right) d_{k}^{S}}\right) I_{k}^{p} \\
& =-\sum_{k=1}^{n} \omega_{k}\left(1-\frac{(p-1)}{p} \frac{\sum_{j=1}^{n} \frac{\beta_{k j} \Lambda_{k}}{d_{k}^{S}}+\frac{p \Lambda_{k}^{2} \sigma_{k}^{2}}{2\left(d_{k}^{S}\right)^{2}}}{d_{k}^{I}+\gamma_{k}+\epsilon_{k}}\right)<-\sum_{k=1}^{n} \omega_{k} \frac{1}{p} I_{k}^{p},
\end{aligned}
$$

hence

$$
\begin{aligned}
L V \leq & -\sum_{k=1}^{n} a_{k}\left\{p d_{k}^{S}-\left[(p-1) \frac{\sum_{j=1}^{n} \beta_{k j} \Lambda_{k}}{d_{k}^{S}}+\frac{(p-1)(p-2) \sigma_{k}^{2} \Lambda_{k}^{2}}{2\left(d_{k}^{S}\right)^{2}}\right] \varepsilon\right\}\left(\frac{\Lambda_{k}}{d_{k}^{S}}-S_{k}\right)^{p} \\
& -\frac{1}{p} \sum_{k=1}^{n} \omega_{k}\left[I_{k}^{p}-\frac{\sum_{j=1}^{n} \beta_{k j} \Lambda_{k}}{d_{k}^{S}\left(d_{k}^{I}+\gamma_{k}+\epsilon_{k}\right)} I_{j}^{p}\right]+\sum_{k=1}^{n}\left[a_{k} \frac{(p-1) \sigma_{k}^{2} \Lambda_{k}^{2}}{\left(d_{k}^{S}\right)^{2}} \varepsilon^{\frac{2-p}{2}}+b_{k} \gamma_{k} \varepsilon^{1-p}\right] I_{k}^{p} \\
& +\sum_{k=1}^{n} a_{k} \frac{\sum_{j=1}^{n} \beta_{k j} \Lambda_{k}\left(\Lambda_{j}\right)^{p}}{d_{k}^{S}\left(d_{j}^{S}\right)^{p}} \varepsilon^{1-p}-\sum_{k=1}^{n} b_{k}\left(p\left(d_{k}^{R}+\delta_{k}\right)-(p-1) \varepsilon \gamma_{k}\right) R_{k}^{p}
\end{aligned}
$$


Note that

$$
\begin{aligned}
& \sum_{k=1}^{n} \omega_{k} I_{k}^{p}-\sum_{k=1}^{n} \omega_{k} \sum_{j=1}^{n} \frac{\beta_{k j} \Lambda_{k}}{d_{k}^{S}\left(d_{k}^{I}+\gamma_{k}+\epsilon_{k}\right)} I_{j}^{p} \\
& \quad=\left(\omega_{1}, \omega_{2}, \ldots, \omega_{n}\right)\left(\begin{array}{cccc}
1-\frac{\beta_{11} S_{1}^{0}}{d_{1}^{I}+\gamma_{1}+\epsilon_{1}} & -\frac{\beta_{11} S_{1}^{0}}{d_{1}^{I}+\gamma_{1}+\epsilon_{1}} & \cdots & -\frac{\beta_{1 n} S_{1}^{0}}{d_{1}^{I}+\gamma_{1}+\epsilon_{1}} \\
-\frac{\beta_{11} S_{2}^{0}}{d_{2}^{I}+\gamma_{2}+\epsilon_{2}} & 1-\frac{\beta_{11} S_{2}^{0}}{d_{2}^{I}+\gamma_{2}+\epsilon_{2}} & \cdots & -\frac{\beta_{1 n} S_{2}^{0}}{d_{2}^{I}+\gamma_{2}+\epsilon_{2}} \\
\vdots & \vdots & \ddots & \vdots \\
-\frac{\beta_{n 1} S_{n}^{0}}{d_{n}^{I}+\gamma_{n}+\epsilon_{n}} & -\frac{\beta_{n 1} S_{n}^{0}}{d_{n}^{I}+\gamma_{n}+\epsilon_{n}} & \cdots & 1-\frac{\beta_{n n} S_{n}^{0}}{d_{n}^{I}+\gamma_{n}+\epsilon_{n}}
\end{array}\right)\left(\begin{array}{c}
I_{1}^{p} \\
I_{2}^{p} \\
\vdots \\
I_{n}^{p}
\end{array}\right) \\
& \quad=\omega\left(E_{n}-M_{0}\right) I^{p} \\
& =\left(1-\rho\left(M_{0}\right)\right)\left(\omega_{1}, \omega_{2}, \ldots, \omega_{n}\right) I^{p},
\end{aligned}
$$

where $I^{p}=\left(I_{1}^{p}, I_{2}^{p}, \ldots, I_{n}^{p}\right)^{T}$. Thus

$$
\begin{aligned}
L V \leq & -\sum_{k=1}^{n} a_{k}\left\{p d_{k}^{S}-\left[(p-1) \frac{\sum_{j=1}^{n} \beta_{k j} \Lambda_{k}}{d_{k}^{S}}+\frac{(p-1)(p-2) \sigma_{k}^{2} \Lambda_{k}^{2}}{2\left(d_{k}^{S}\right)^{2}}\right] \varepsilon\right\}\left(\frac{\Lambda_{k}}{d_{k}^{S}}-S_{k}\right)^{p} \\
& -\frac{1}{p}\left(1-\rho\left(M_{0}\right)\right)\left(\omega_{1}, \omega_{2}, \ldots, \omega_{n}\right) I^{p}+\sum_{k=1}^{n}\left[a_{k} \frac{(p-1) \sigma_{k}^{2} \Lambda_{k}^{2}}{\left(d_{k}^{S}\right)^{2}} \varepsilon^{\frac{2-p}{2}}+b_{k} \gamma_{k} \varepsilon^{1-p}\right] I_{k}^{p} \\
& +\sum_{k=1}^{n} a_{k} \frac{\sum_{j=1}^{n} \beta_{k j} \Lambda_{k}\left(\Lambda_{j}\right)^{p}}{d_{k}^{S}\left(d_{j}^{S}\right)^{p}} \varepsilon^{1-p}-\sum_{k=1}^{n} b_{k}\left(p\left(d_{k}^{R}+\delta_{k}\right)-(p-1) \varepsilon \gamma_{k}\right) R_{k}^{p} .
\end{aligned}
$$

An $\varepsilon$ can be chosen small enough so that both the coefficients of $\left(\frac{\Lambda_{k}}{d_{k}^{s}}-S_{k}\right)^{p}$ and the coefficients of $R_{k}^{p}$ are negative, and we can also choose sufficiently small $a_{k}$ and $b_{k}$ such that

$$
\begin{aligned}
& -\frac{1}{p}\left(1-\rho\left(M_{0}\right)\right)\left(\omega_{1}, \omega_{2}, \ldots, \omega_{n}\right) I^{p}+\sum_{k=1}^{n}\left[a_{k} \frac{(p-1) \sigma_{k}^{2} \Lambda_{k}^{2}}{\left(d_{k}^{S}\right)^{2}} \varepsilon^{\frac{2-p}{2}}+b_{k} \gamma_{k} \varepsilon^{1-p}\right] I_{k}^{p} \\
& \quad+\sum_{k=1}^{n} a_{k} \frac{\sum_{j=1}^{n} \beta_{k j} \Lambda_{k}\left(\Lambda_{j}\right)^{p}}{d_{k}^{S}\left(d_{j}^{S}\right)^{p}} \varepsilon^{1-p}
\end{aligned}
$$

is negative. Consequently, $L V$ is negative-definite. Therefore, we conclude that under the condition $\mathscr{R}_{0} \leq 1$, the equilibrium $P_{0}$ of (1.4) is stochastically asymptotically stable at large.

When $p=2$, we have a corollary of Theorems 3.4 and 3.6.

Corollary 3.7 Assume $B=\left(\beta_{k j}\right)_{n \times n}$ is irreducible. If $\mathscr{R}_{0}=\rho\left(M_{0}\right) \leq 1$ and $\left(d_{k}^{I}+\gamma_{k}+\epsilon_{k}\right)-$ $\sum_{j=1}^{n} \frac{\beta_{k j} \Lambda_{k}}{d_{k}^{S}}>\frac{p \Lambda_{k}^{2} \sigma_{k}^{2}}{2\left(d_{k}^{S}\right)^{2}}$ hold, then the disease-free equilibrium $P_{0}$ of (1.4) is globally asymptotically stable.

Theorem 3.8 If $2 \sigma_{k}^{2}\left(d_{k}^{S}-\sum_{j=1, j \neq k}^{n} \beta_{k j} \frac{\Lambda_{k}}{d_{k}^{S}}\right)>\beta_{k k}^{2}$, then the disease-free equilibrium $P_{0}$ is almost surely exponentially stable in $\Gamma^{*}$. 
Proof Let $\left(S_{0}, I_{0}, R_{0}\right) \in \Gamma^{*}$. By Theorem 3.2, the solution of (1.4) belongs to the set $\Gamma^{*}$. Define

$$
V_{4}=\ln \left[\sum_{k=1}^{n}\left(\frac{\Lambda_{k}}{d_{k}^{S}}-S_{k}+I_{k}+R_{k}\right)\right] .
$$

Applying Ito's formula [24], we obtain

$$
\begin{aligned}
L V_{4}= & \sum_{k=1}^{n}\left[\frac{\partial V_{4}}{\partial S_{k}} \mathrm{~d} S_{k}+\frac{\partial V_{4}}{\partial I_{k}} \mathrm{~d} I_{k}+\frac{\partial V_{4}}{\partial R_{k}} \mathrm{~d} R_{k}\right] \\
& +\sum_{k=1}^{n}\left[\frac{\partial^{2} V_{4}}{\partial S_{k}^{2}} \mathrm{~d} S_{k} \mathrm{~d} S_{k}+\frac{\partial^{2} V_{4}}{\partial I_{k}^{2}} \mathrm{~d} I_{k} \mathrm{~d} I_{k}+\frac{\partial^{2} V_{4}}{\partial R_{k}^{2}} \mathrm{~d} R_{k} \mathrm{~d} R_{k}\right] \\
& +\sum_{k=1}^{n}\left[\frac{\partial^{2} V_{4}}{\partial S_{k} \partial I_{k}} \mathrm{~d} S_{k} \mathrm{~d} I_{k}+\frac{\partial^{2} V_{4}}{\partial S_{k} \partial R_{k}} \mathrm{~d} S_{k} \mathrm{~d} R_{k}+\frac{\partial^{2} V_{4}}{\partial I_{k} \partial R_{k}} \mathrm{~d} I_{k} \mathrm{~d} R_{k}\right] \\
= & \frac{1}{\sum_{k=1}^{n}\left(\frac{\Lambda_{k}}{d_{k}^{S}}-S_{k}+I_{k}+R_{k}\right)}\left(\sum_{k=1}^{n}\left(-\mathrm{d} S_{k}+\mathrm{d} I_{k}+\mathrm{d} R_{k}\right)\right) \\
& -\frac{1}{2\left(\sum_{k=1}^{n}\left(\frac{\Lambda_{k}}{d_{k}^{S}}-S_{k}+I_{k}+R_{k}\right)\right)^{2}}\left(\sum_{k=1}^{n}\left(\mathrm{~d} S_{k} \mathrm{~d} S_{k}+\mathrm{d} I_{k} \mathrm{~d} I_{k}+\mathrm{d} R_{k} \mathrm{~d} R_{k}\right)\right) \\
& +\frac{1}{\left(\sum_{k=1}^{n}\left(\frac{\Lambda_{k}}{d_{k}^{S}}-S_{k}+I_{k}+R_{k}\right)\right)^{2}}\left(\sum_{k=1}^{n}\left(\mathrm{~d} S_{k} \mathrm{~d} I_{k}+\mathrm{d} S_{k} \mathrm{~d} R_{k}-\mathrm{d} I_{k} \mathrm{~d} R_{k}\right)\right)
\end{aligned}
$$

where $\mathrm{d} B \mathrm{~d} B=\mathrm{d} t$ and $\mathrm{d} B \mathrm{~d} t=\mathrm{d} t \mathrm{~d} B=0$. Then

$$
\begin{aligned}
\mathrm{d} S_{k} \mathrm{~d} S_{k} & =\mathrm{d} I_{k} \mathrm{~d} I_{k}=-\mathrm{d} S_{k} \mathrm{~d} I_{k}=\sigma_{k}^{2}\left(\frac{S_{k} I_{k}}{1+\alpha_{k} I_{k}}\right)^{2} \mathrm{~d} t, \quad \mathrm{~d} S_{k} \mathrm{~d} R_{k}=\mathrm{d} I_{k} \mathrm{~d} R_{k}=\mathrm{d} R_{k} \mathrm{~d} R_{k}=0 \\
L V_{4}= & \frac{1}{\sum_{i=1}^{n}\left(\frac{\Lambda_{i}}{d_{i}^{S}}-S_{i}+I_{i}+R_{i}\right)}\left[\sum_{k=1}^{n}\left(-\Lambda_{k}+d_{k}^{S} S_{k}+\sum_{j=1}^{n} \frac{\beta_{k j} S_{k} I_{j}}{1+\alpha_{k} I_{j}}-\delta_{k} R_{k}\right)\right] \mathrm{d} t \\
& +\frac{1}{\sum_{i=1}^{n}\left(\frac{\Lambda_{j}}{d_{i}^{S}}-S_{i}+I_{i}+R_{i}\right)}\left[\sum_{k=1}^{n}\left(\sum_{j=1}^{n} \frac{\beta_{k j} S_{k} I_{j}}{1+\alpha_{k} I_{j}}-\left(d_{k}^{I}+\gamma_{k}\right) I_{k}\right)\right] \mathrm{d} t \\
& +\frac{1}{\sum_{i=1}^{n}\left(\frac{\Lambda_{i}}{d_{i}^{S}}-S_{i}+I_{i}+R_{i}\right)}\left[\sum_{k=1}^{n}\left(\gamma_{k} I_{k}-\left(d_{k}^{R}+\delta_{k}\right) R_{k}\right)\right] \mathrm{d} t \\
& -\sum_{k=1}^{n} 2 \sigma_{k}^{2}\left(\frac{S_{k} I_{k}}{\left(1+\alpha_{k} I_{k}\right) \sum_{i=1}^{n}\left(\frac{\Lambda_{i}}{d_{i}^{S}}-S_{i}+I_{i}+R_{i}\right)}\right)^{2} \mathrm{~d} t \\
& +\sum_{k=1}^{n} \frac{2 \sigma_{k} S_{k} I_{k}}{\left(1+\alpha_{k} I_{k}\right) \sum_{i=1}^{n}\left(\frac{\Lambda_{i}}{d_{i}^{S}}-S_{i}+I_{i}+R_{i}\right)} \mathrm{d} B .
\end{aligned}
$$




$$
\begin{aligned}
& \text { Let } M_{k}=\frac{S_{k} I_{k}}{\left(1+\alpha_{k} I_{k}\right) \sum_{i=1}^{n}\left(\frac{\Lambda_{i}}{d_{i}^{S}}-S_{i}+I_{i}+R_{i}\right)} \text {, we have } \\
& L V_{4}=\sum_{k=1}^{n}\left[-2 \sigma_{k}^{2} M_{k}^{2}+2 \beta_{k k} M_{k}-\frac{d_{k}^{S}\left(\frac{\Lambda_{k}}{d_{k}^{S}}-S_{k}\right)+d_{k}^{I} I_{k}+d_{k}^{R} R_{k}}{\sum_{i=1}^{n}\left(\frac{\Lambda_{i}}{d_{i}^{S}}-S_{i}+I_{i}+R_{i}\right)}\right] \mathrm{d} t \\
& +\frac{1}{\sum_{i=1}^{n}\left(\frac{\Lambda_{i}}{d_{i}^{S}}-S_{i}+I_{i}+R_{i}\right)} \sum_{k=1}^{n} \sum_{j=1, j \neq k}^{n} \frac{\beta_{k j} S_{k} I_{j}}{1+\alpha_{k} I_{j}} \mathrm{~d} t+2 \sigma_{k} M_{k} \mathrm{~d} B \\
& \leq \sum_{k=1}^{n}\left[-2 \sigma_{k}^{2} M_{k}^{2}+2 \beta_{k k} M_{k}-\frac{d_{k}^{S}\left(\frac{\Lambda_{k}}{d_{k}^{S}}-S_{k}+I_{k}+R_{k}\right)}{\sum_{i=1}^{n}\left(\frac{\Lambda_{i}}{d_{i}^{S}}-S_{i}+I_{i}+R_{i}\right)}\right] \mathrm{d} t \\
& +\frac{1}{\sum_{i=1}^{n}\left(\frac{\Lambda_{i}}{d_{i}^{S}}-S_{i}+I_{i}+R_{i}\right)} \sum_{k=1}^{n} \sum_{j=1, j \neq k}^{n} \frac{\beta_{k j} S_{k} I_{j}}{1+\alpha_{k} I_{j}} \mathrm{~d} t+\sum_{k=1}^{n} 2 \sigma_{k} M_{k} \mathrm{~d} B, \\
& \sum_{k=1}^{n} d_{k}^{S}\left(\frac{\Lambda_{k}}{d_{k}^{S}}-S_{k}+I_{k}+R_{k}\right) \\
& \leq \sum_{k=1}^{n} d_{k}^{S} \sum_{k=1}^{n}\left(\frac{\Lambda_{k}}{d_{k}^{S}}-S_{k}+I_{k}+R_{k}\right) \frac{1}{\sum_{i=1}^{n}\left(\frac{\Lambda_{i}}{d_{i}^{S}}-S_{i}+I_{i}+R_{i}\right)} \sum_{k=1}^{n} \sum_{j=1, j \neq k}^{n} \frac{\beta_{k j} S_{k} I_{j}}{1+\alpha_{k} I_{j}} \\
& \leq \sum_{k=1}^{n} \sum_{j=1, j \neq k}^{n} \beta_{k j} \frac{\Lambda_{k}}{d_{k}^{S}} \\
& L V_{4} \leq \sum_{k=1}^{n}\left(-2 \sigma_{k}^{2} M_{k}^{2}+2 \beta_{k k} M_{k}-d_{k}^{S}+\sum_{j=1, j \neq k}^{n} \beta_{k j} \frac{\Lambda_{k}}{d_{k}^{S}}\right) \mathrm{d} t+\sum_{k=1}^{n} 2 \sigma_{k} M_{k} \mathrm{~d} B \\
& =\sum_{k=1}^{n}\left(-2 \sigma_{k}^{2}\left(M_{k}-\frac{\beta_{k k}}{2 \sigma_{k}^{2}}\right)^{2}+\frac{\beta_{k k}^{2}-2 d_{k}^{S} \sigma_{k}^{2}+2 \sigma_{k}^{2} \sum_{j=1, j \neq k}^{n} \beta_{k j} \frac{\Lambda_{k}}{d_{k}^{S}}}{2 \sigma_{k}^{2}}\right) \mathrm{d} t \\
& +\sum_{k=1}^{n} 2 \sigma_{k} M_{k} \mathrm{~d} B
\end{aligned}
$$

we deduce that

$$
\mathrm{d} V_{4} \leq \sum_{k=1}^{n} \frac{\beta_{k k}^{2}-2 d_{k}^{S} \sigma_{k}^{2}+2 \sigma_{k}^{2} \sum_{j=1, j \neq k}^{n} \beta_{k j} \frac{\Lambda_{k}}{d_{k}^{S}}}{2 \sigma_{k}^{2}} \mathrm{~d} t+\sum_{k=1}^{n} 2 \sigma_{k} M_{k} \mathrm{~d} B
$$

and by integration we get

$$
\begin{aligned}
\ln \left[\sum_{k=1}^{n}\left(\frac{\Lambda_{k}}{d_{k}^{S}}-S_{k}+I_{k}+R_{k}\right)\right] \\
\leq \ln \left[\sum_{k=1}^{n}\left(\frac{\Lambda_{k}}{d_{k}^{S}}-S_{k}^{0}+I_{k}^{0}+R_{k}^{0}\right)\right]+\sum_{k=1}^{n} \frac{\beta_{k k}^{2}-2 d_{k}^{S} \sigma_{k}^{2}+2 \sigma_{k}^{2} \sum_{j=1, j \neq k}^{n} \beta_{k j} \frac{\Lambda_{k}}{d_{k}^{S}}}{2 \sigma_{k}^{2}} t \\
\quad+\int_{0}^{t} \sum_{k=1}^{n} 2 \sigma_{k} M_{k}(s) \mathrm{d} B(s) .
\end{aligned}
$$


From Theorem 3.2, we obtain $\int_{0}^{t} \sum_{k=1}^{n} M_{k}(s) \mathrm{d} B(s)=\int_{0}^{t} \sum_{k=1}^{n} M_{k}^{2}(s) \mathrm{d} B(s) \leq C t$. According to the strong law of large number [25], we deduce

$$
\lim _{t \rightarrow \infty} \frac{1}{t} \int_{0}^{t} \sum_{k=1}^{n} M_{k}(s) \mathrm{d} B(s)=0 \quad \text { a.s. }
$$

Therefore, from (3.26) and (3.27) we conclude that

$$
\limsup _{t \rightarrow \infty} \ln \left[\sum_{k=1}^{n}\left(\frac{\Lambda_{k}}{d_{k}^{S}}-S_{k}+I_{k}+R_{k}\right)\right] \leq \sum_{k=1}^{n}\left(\beta_{k k}^{2}-2 d_{k}^{S} \sigma_{k}^{2}+2 \sigma_{k}^{2} \sum_{j=1, j \neq k}^{n} \beta_{k j} \frac{\Lambda_{k}}{d_{k}^{S}}\right)<0 .
$$

\section{Numerical simulations}

Using the Matlab software, we calculate the equilibrium of (1.3) when $n=2$. In this case, we have

$$
M_{0}=\left[\begin{array}{cc}
\frac{\beta_{11} S_{1}^{0}}{d_{1}^{I}+\epsilon_{1}+\gamma_{1}} & \frac{\beta_{12} S_{1}^{0}}{d_{1}^{I}+\epsilon_{1}+\gamma_{1}} \\
\frac{\beta_{21} S_{2}^{0}}{d_{2}^{I}+\epsilon_{2}+\gamma_{2}} & \frac{\beta_{22} S_{2}^{0}}{d_{2}^{I}+\epsilon_{2}+\gamma_{2}}
\end{array}\right]:=\left[\begin{array}{ll}
\beta_{11} K_{1} & \beta_{12} K_{1} \\
\beta_{21} K_{2} & \beta_{22} K_{2}
\end{array}\right]
$$

and

$$
\mathscr{R}_{0}=\rho\left(M_{0}\right)=\frac{\beta_{11} K_{1}+\beta_{22} K_{2}+\sqrt{\left(\beta_{11} K_{1}-\beta_{22} K_{2}\right)^{2}+4 \beta_{12} \beta_{21} K_{1} K_{2}}}{2},
$$

where $K_{1}=\frac{S_{1}^{0}}{d_{1}^{I}+\epsilon_{1}+\gamma_{1}}, K_{2}=\frac{S_{2}^{0}}{d_{2}^{I}+\epsilon_{2}+\gamma_{2}}$.

If the system parameters are given by

$$
\begin{aligned}
& \Lambda_{1}=2.5, \quad \beta_{11}=0.50, \quad \beta_{12}=0.10, \quad \alpha_{1}^{S}=0.2, \quad d_{1}^{S}=0.60, \quad d_{1}^{I}=0.85 \text {, } \\
& d_{1}^{R}=0.86, \quad \epsilon_{1}=0.4, \quad \delta_{1}=0.5, \quad \gamma_{1}=0.45, \\
& \Lambda_{2}=3.5, \quad \beta_{21}=0.12, \quad \beta_{22}=0.45, \quad \alpha_{1}^{S}=0.3, \quad d_{2}^{S}=0.70, \quad d_{2}^{I}=0.78 \text {, } \\
& d_{2}^{R}=0.83, \quad \epsilon_{2}=0.5, \quad \delta_{2}=0.1, \quad \gamma_{2}=0.5 \text {, }
\end{aligned}
$$

then it is easy to compute that $\mathscr{R}_{0}=1.5331>1$. The computation shows that $P^{*}=$ $\left(S_{1}^{*}, I_{1}^{*}, R_{1}^{*}, S_{2}^{*}, I_{2}^{*}, R_{2}^{*}\right)=(0.5162,0.1708,2.8465,0.8733,0.4695,4.2584)$. The computing result is in good agreement with the statement of Corollary 2.5 .

Next, we simulate the solutions of (1.4). Discretizing the system (1.4) for $t=0, \Delta t, 2 \Delta t$, $\ldots, n \triangle t$, and $k=1,2$, we have

$$
\left\{\begin{aligned}
S_{k, i+1}= & S_{k, i}+\left(\Lambda_{k}-\frac{\beta_{k 1} S_{k, i} I_{1, i}}{1+\alpha_{k} I_{1, i}}-\frac{\beta_{k 2} S_{k, i} I_{2, i}}{1+\alpha_{k} I_{2, i}}-d_{k}^{S} S_{k, i}+\delta_{k} R_{k, i}\right) \Delta t \\
& -\sigma_{k} \frac{\beta_{k k} S_{k, i} I_{k, i}}{1+\alpha_{k} I_{k, i}} \sqrt{\Delta t} \varepsilon_{k, i}, \\
I_{k, i+1}= & I_{k, i}+\left(\frac{\beta_{k 1} S_{k, i} I_{1, i}}{1+\alpha_{k} I_{1, i}}+\frac{\beta_{k 2} S_{k, i} I_{2, i}}{1+\alpha_{k} I_{2, i}}-\left(d_{k}^{I}+\gamma_{k}+\epsilon_{k}\right) I_{k, i}\right) \Delta t+\sigma_{k} \frac{\beta_{k k} S_{k, i} I_{k, i}}{1+\alpha_{k} I_{k, i}} \sqrt{\triangle t} \varepsilon_{k, i}, \\
R_{k, i+1}= & R_{k, i}+\left(\gamma_{k} I_{k, i}+\left(d_{k}^{R}+\delta_{k}\right) R_{k, i}\right) \Delta t,
\end{aligned}\right.
$$

where the time increment $\Delta t>0$, and $\varepsilon_{k, i}$ are $N(0,1)$-distributed independent random variables, which can be generated numerically by pseudo-random number generators. Moreover, we choose $S_{1}(0)=2.5, I_{1}(0)=1.3, R_{1}(0)=0.75, S_{2}(0)=5.3, I_{2}(0)=1.5, R_{2}(0)=$ 

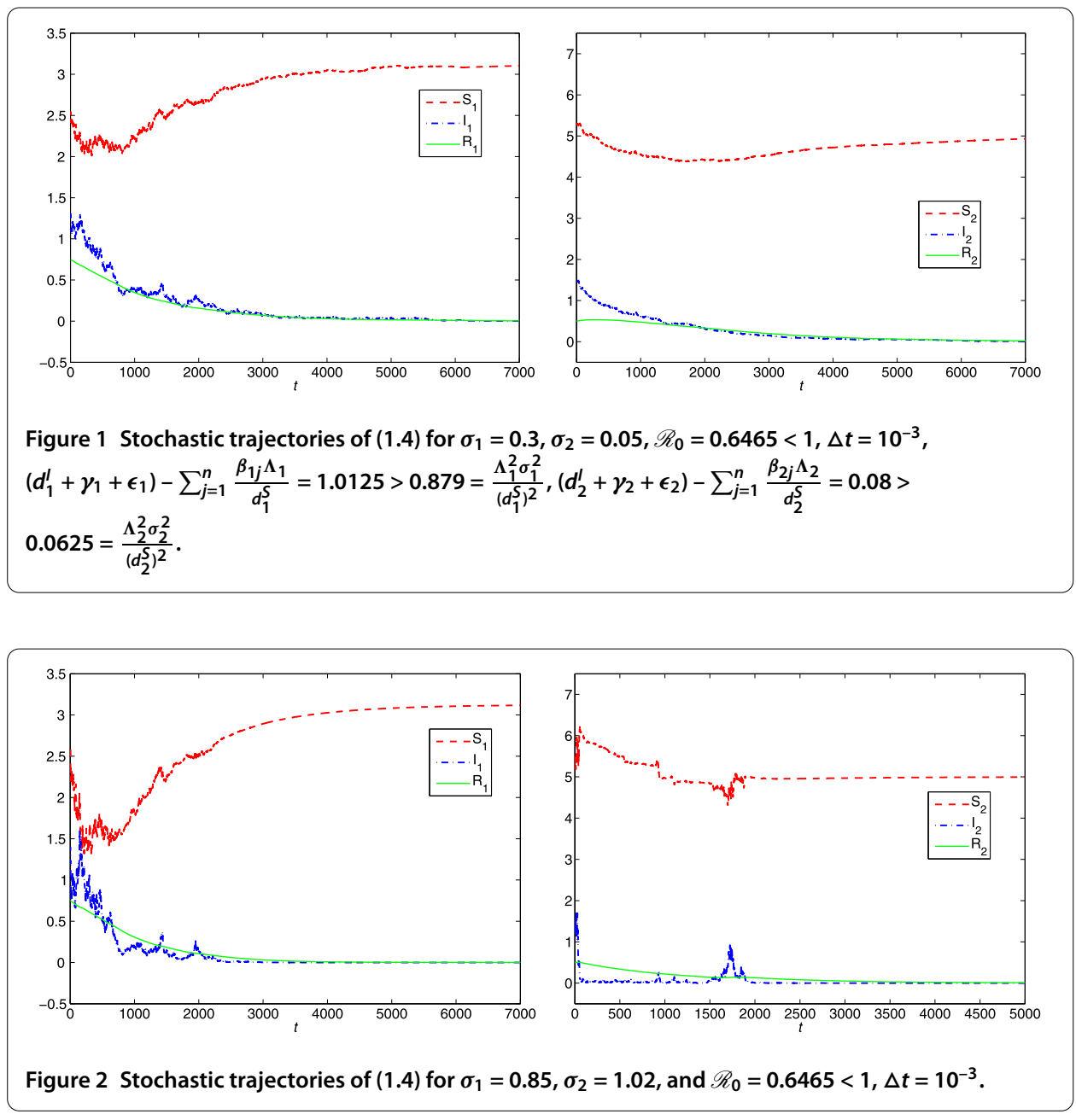

0.5 as the initial values. We state that the initial values in every example are identical with the values mentioned above. The simulation on the asymptotic stability of the equilibrium $P_{0}$ of (1.4) is shown in Figure 1 for $\sigma_{1}=0.3, \sigma_{2}=0.05$. The system parameters are given by

$$
\begin{aligned}
& \Lambda_{1}=2.5, \quad \beta_{11}=0.10, \quad \beta_{12}=0.12, \quad \alpha_{1}^{S}=0.2, \quad d_{1}^{S}=0.80, \quad d_{1}^{I}=0.85, \\
& d_{1}^{R}=0.86, \quad \epsilon_{1}=0.4, \quad \delta_{1}=0.5, \quad \gamma_{1}=0.45 \text {, } \\
& \Lambda_{2}=3.5, \quad \beta_{21}=0.21, \quad \beta_{22}=0.13, \quad \alpha_{1}^{S}=0.3, \quad d_{2}^{S}=0.70, \quad d_{2}^{I}=0.78, \\
& d_{2}^{R}=0.83, \quad \epsilon_{2}=0.5, \quad \delta_{2}=0.1, \quad \gamma_{2}=0.5,
\end{aligned}
$$

then we obtain $\mathscr{R}_{0}=0.6465<1$. By a simple computation, $P_{0}=\left(S_{1}^{0}, I_{1}^{0}, R_{1}^{0}, S_{2}^{0}, I_{2}^{0}, R_{2}^{0}\right)=$ $(3.125,0,0,5,0,0)$. It must be notified that we adopt the same coefficient parameters as shown above in the examples of Figures 2-5, and this means that the calculated reproduction numbers are the same.

If we adapt the first group of parameters for system (1.4), that is, the parameters are taken as follows:

$$
\Lambda_{1}=2.5, \quad \beta_{11}=0.50, \quad \beta_{12}=0.10, \quad \alpha_{1}^{S}=0.2, \quad d_{1}^{S}=0.60, \quad d_{1}^{I}=0.85,
$$



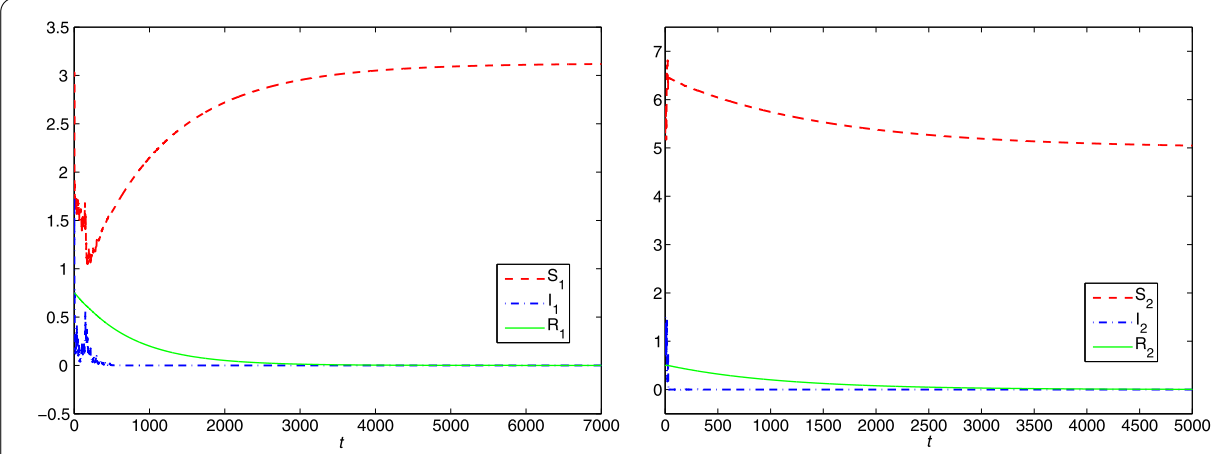

Figure 3 Stochastic trajectories of (1.4) for $\sigma_{1}=3.75, \sigma_{2}=2.72$, and $\mathscr{R}_{0}=0.6465<1, \Delta t=10^{-3}$.
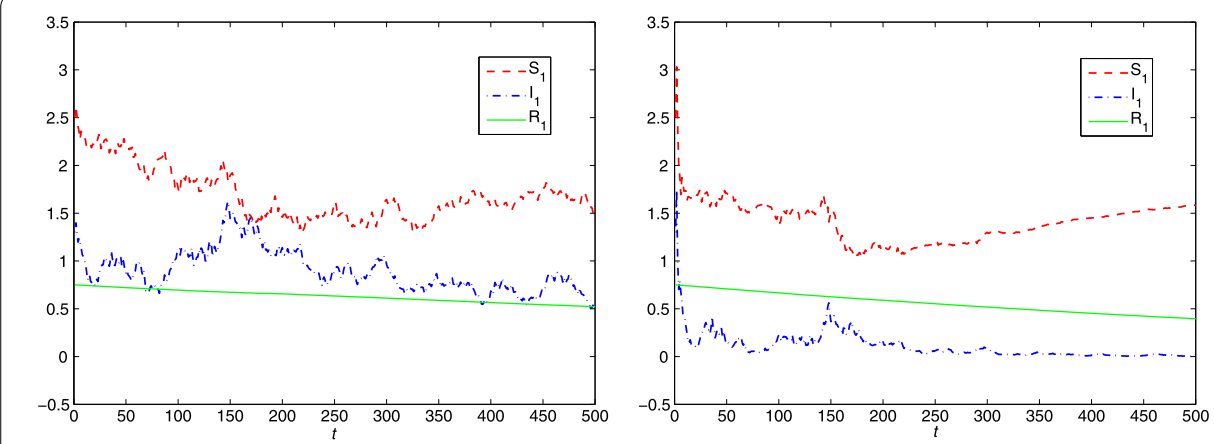

Figure 4 Stochastic trajectories $S_{1}(t), l_{1}(t), R_{1}(t)$ when specifying the intensities of the Brownian motions as $\sigma_{1}=0.85, \sigma_{2}=1.02$ (left) and $\sigma_{1}=3.75, \sigma_{2}=2.72$ (right), respectively, and $t \in[0,500]$.
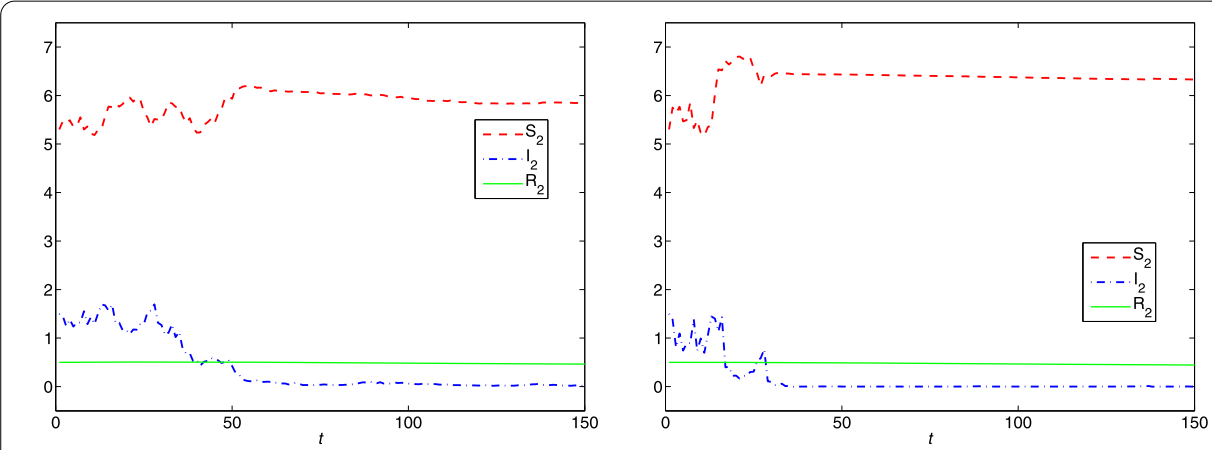

Figure 5 Stochastic trajectories $S_{2}(t), I_{2}(t), R_{2}(t)$ when specifying the intensities of the Brownian motions as $\sigma_{1}=0.85, \sigma_{2}=1.02$ (left) and $\sigma_{1}=3.75, \sigma_{2}=2.72$ (right), respectively, and $t \in[0,500]$.

$$
\begin{array}{ll}
d_{1}^{R}=0.86, \quad \epsilon_{1}=0.4, & \delta_{1}=0.5, \quad \gamma_{1}=0.45, \\
\Lambda_{2}=3.5, \quad \beta_{21}=0.12, & \beta_{22}=0.45, \quad \alpha_{1}^{S}=0.3, \quad d_{2}^{S}=0.70, \\
d_{2}^{I}=0.78, \quad d_{2}^{R}=0.83, \quad \epsilon_{2}=0.5, \quad \delta_{2}=0.1, \quad \gamma_{2}=0.5,
\end{array}
$$

then the calculated reproduction number is still $\mathscr{R}_{0}=1.5331>1$, but if we change the noise intensity to $\sigma_{1}=2.2, \sigma_{2}=1.9$, the corresponding simulations are shown in Figure 6 . 


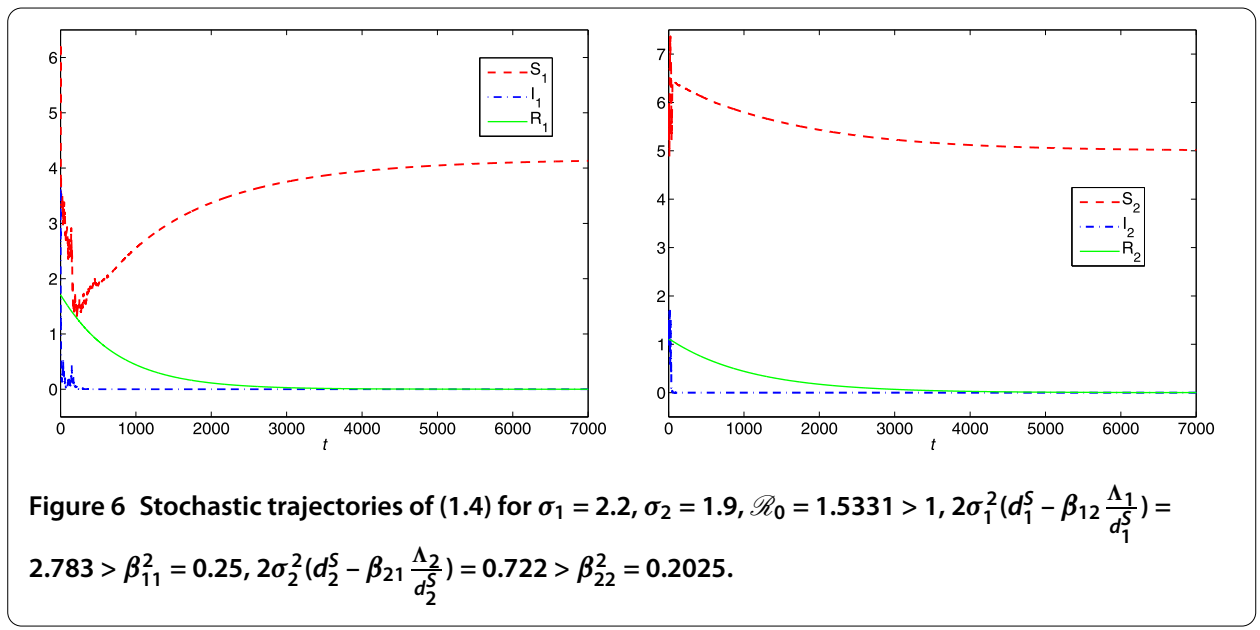

Figure 1 corresponds to $\sigma_{1}=0.3, \sigma_{2}=0.05$, and a simple check shows that the intensity and system parameters satisfy the condition of Corollary 3.7. We can therefore conclude, by Corollary 3.7, that the equilibrium $P_{0}$ of (1.4) is asymptotically stable. There exists good agreement between the mathematical results and computer simulations in Figure 1. Figure 2 corresponds to $\sigma_{1}=0.85, \sigma_{2}=1.02$, and the comparison of Figures 1 (left) and 2 (left) suggests that the fluctuations of the curves increase as the noise level increases. The trajectories of Figures 1 (right) and 2 (right) follow the same regularity. So does the comparison of Figures 2 and 3. Note that the condition of Corollary 3.7 is sufficient but not necessary. In other words, if the condition of Corollary 3.7 is not satisfied, the system (1.4) could be stable. For example, both $\sigma_{1}=0.85, \sigma_{2}=1.02$ and $\sigma_{1}=3.75, \sigma_{2}=2.72$ do not obey the condition of Corollary 3.7, but we can see from Figures 2 and 3 that the equilibrium $P_{0}$ remains asymptotically stable. In order to observe the influences of the noise intensity of the stochastic system (1.4) much clearer, we re-draw the solution curves of system (1.4) in Figure 4 and Figure 5 when the intensities of the Brownian motions are specified as $\sigma_{1}=0.85, \sigma_{2}=1.02$ or $\sigma_{1}=3.75, \sigma_{2}=2.72$, respectively, and $t \in[0,500]$. Analyzing the curves in Figures 4 and 5, they indicate that the higher the values of the intensities of the Brownian motions are, the more violent the fluctuations of solution curves are, and the shorter time solution curves attain to the equilibrium $P_{0}$. Our numerical simulations are in good agreement with the theoretical results derived from complicated analysis methods.

\section{Conclusion}

In this paper, we propose deterministic and stochastic multi-group SIRS models with a saturated incidence rate. Making use of the Perron-Frobenius theorem, the La Salle invariance principle, and the Lyapunov function analysis method, we obtain the theoretical results describing the dynamical behavior of these epidemic models. These theories are the further developments of the study by Lahrouz et al. [10] for single-group SIRS models with a saturated incidence rate. As discussed in Section 3, there exists an important threshold $\mathscr{R}_{0}$ determining the persistence and the extinction of disease for the deterministic model. As for the stochastic model, some sufficient criterions for stochastic asymptotical stability of the equilibrium $P_{0}$ are established via stochastic analysis techniques. It is further found that the $p$ th moment exponential stability of $P_{0}$ depends on the threshold $\mathscr{R}_{0}$, the magnitude of the intensity of noise, and the parameters of (1.4) except the param- 
eter $\alpha_{k}$, which measures the saturation effect. However, it must be noted that the almost surely exponential stability depends on the intensity of noise and the parameters of (1.4) only, while the threshold $\mathscr{R}_{0}$ has no effect at all, even when $\mathscr{R}_{0}>1,(1.4)$ remains almost surely exponentially stable.

\section{Competing interests}

The authors declare that they have no competing interests.

\section{Authors' contributions}

All authors contributed equally to the writing of this paper. All authors read and approved the final manuscript.

\section{Author details}

'School of Mathematics, Changchun Normal University, Changchun, 130032, P.R. China. ${ }^{2}$ School of Mathematical Sciences, Harbin Normal University, Harbin, 150500, P.R. China. ${ }^{3}$ College of Mathematics, Jilin University, Changchun, 130012, P.R. China.

\section{Acknowledgements}

This project is supported by the China Postdoctoral Science Foundation (Grant No. 2014M551168), the Natural Science Foundation of Heilongjiang Province of China (Grant No. A201410) and Scientific Research Foundation of Jilin Provincial Education Department (Grant No. 2015356).

Received: 11 June 2015 Accepted: 31 August 2015 Published online: 24 October 2015

\section{References}

1. Shaikhet, L: Stability of predator-prey model with aftereffect by stochastic perturbation. Stab. Control: Theory Appl. 1, 3-13 (1998)

2. Shaikhet, L: Stability of a positive point of equilibrium of one nonlinear system with aftereffect and stochastic perturbations. Dyn. Syst. Appl. 17, 235-253 (2008)

3. Guo, H, Li, MY, Shuai, Z: Global stability of the endemic equilibrium of multigroup SIR epidemic models. Can. Appl. Math. Q. 14, 259-284 (2006)

4. Lajmanovich, A, Yorke, JA: A deterministic model for gonorrhea in a nonhomogeneous population. Math. Biosci. 28 221-236 (1976)

5. Kuniya, T: Global stability of a multi-group SVIR epidemic model. Nonlinear Anal., Real World Appl. 14, 1135-1143 (2013)

6. Muroya, Y, Enatsu, Y, Kuniya, T: Global stability for a multi-group SIRS epidemic model with varying population sizes. Nonlinear Anal., Real World Appl. 14, 1693-1704 (2013)

7. Ji, C, Jiang, D, Shi, N: Multigroup SIR epidemic model with stochastic perturbation. Physica A 390, 1747-1762 (2011)

8. Wang, Z, Fan, X, Jiang, F, Li, Q: Dynamics of deterministic and stochastic multi-group MSIRS epidemic models with varying total population size. Adv. Differ. Equ. 2014, 270 (2014)

9. Capasso, V, Serio, G: A generalization of the Kermack-McKendrick deterministic epidemic model. Math. Biosci. 42, 41-61 (1978)

10. Lahrouz, A, Omari, L, Kiouach, D: Global analysis of a deterministic and stochastic nonlinear SIRS epidemic model. Nonlinear Anal., Model. Control 16, 59-76 (2011)

11. Wang, Z, Fan, X: Global stability of deterministic and stochastic multigroup SEIQR models in computer network. Appl. Math. Model. 37, 8673-8686 (2013)

12. Fan, $X$, Wang, Z: Stability analysis of an SEIR epidemic model with stochastic perturbation and numerical simulation. Int. J. Nonlinear Sci. Numer. Simul. 14, 113-121 (2013)

13. Wang, Z, Gao, R, Fan, X, Han, Q: Stability analysis of multi-group deterministic and stochastic epidemic models with vaccination rate. Chin. Phys. B 23, 090201 (2014)

14. Imhof, L, Walcher, S: Exclusion and persistence in deterministic and stochastic chemostat models. J. Differ. Equ. 217, 26-53 (2005)

15. Dalal, N, Greenhalgh, D, Mao, X: A stochastic model of AIDS and condom use. J. Math. Anal. Appl. 325, 36-53 (2007)

16. Jiang, D, Yu, J, Ji, C, Shi, N: Asymptotic behavior of global positive solution to a stochastic SIR model. Math. Comput. Model. 54, 221-232 (2011)

17. van den Driessche, $P$, Watmough, J: Reproduction numbers and sub-threshold endemic equilibria for compartmental models of disease transmission. Math. Biosci. 180, 29-48 (2002)

18. Berman, A, Plemmons, RJ: Nonnegative Matrices in the Mathematical Sciences. Academic Press, New York (1979)

19. Lasalle, JP: The Stability of Dynamical Systems. Regional Conference Series in Applied Mathematics. SIAM, Philadelphia (1976)

20. Freedman, HI, Ruan, S, Tang, M: Uniform persistence and flows near a closed positively invariant set. J. Dyn. Differ. Equ. 6, 583-600 (1994)

21. Li, MY, Graef, JR, Wang, L, Karsai, J: Global dynamics of a SEIR model with varying total population size. Math. Biosci. 160, 191-213 (1999)

22. Arnold, L: Stochastic Differential Equations: Theory and Applications. Wiley, New York (1972)

23. Mao, X: Stochastic Differential Equations and Applications. Ellis Horwood, Chichester (1997)

24. Øksendal, B: Stochastic Differential Equations: An Introduction with Applications. Springer, Heidelberg (2000)

25. Liptser, R: A strong law of large numbers for local martingales. Stochastics 3, 217-228 (1980) 